\title{
Is Memory Search Governed by Universal Principles or Idiosyncratic Strategies?
}

\author{
M. Karl Healey and Michael J. Kahana \\ University of Pennsylvania
}

\begin{abstract}
Laboratory paradigms have provided an empirical foundation for much of psychological science. Some have argued, however, that such paradigms are highly susceptible to idiosyncratic strategies and that many findings do not reflect fundamental cognitive principles but are instead artifacts of averaging across participants who employ different strategies. We developed a set of techniques to rigorously test the extent to which average data are distorted by such strategy differences and applied these techniques to free recall data from the Penn Electrophysiology of Encoding and Retrieval Study. Recall initiation showed evidence of subgroups: The majority of participants initiated recall from the last item in the list, but one subgroup showed elevated initiation probabilities for items 2 to 4 positions back from the end of the list, and another showed elevated probabilities for the beginning of the list. By contrast, serial position curves and temporal and semantic clustering functions were remarkably consistent, with almost every participant exhibiting a recognizable version of the average function, suggesting that these functions reflect fundamental principles of the memory system. The approach taken here can serve as a model for evaluating the extent to which other laboratory paradigms are influenced by individual differences in strategy use.
\end{abstract}

Keywords: strategy, free recall, episodic memory

Much of psychological research relies on laboratory paradigms that have been designed to measure specific cognitive processes. Each area of research has its own set of laboratory paradigms: For example, psychologists studying perception use detection and discrimination tasks (Chun \& Wolfe, 2001), psychologists studying memory use list recall and recognition tasks (Kahana, 2012), psychologists studying language use word naming and lexical decision tasks (Balota et al., 2007), and those studying decision making use preference and temporal discounting tasks (Loewenstein \& Prelec, 1993). An assumption of such paradigm-centric research is that participants' performance on laboratory tasks reflects the operation of the targeted cognitive processes. However, all but the simplest task is likely to rely on multiple processes. Moreover, many researchers have argued that performing a task involves selecting strategies from a cognitive toolbox to create ad hoc solutions to challenges posed by the task (Gigerenzer, 2008; Gigerenzer, Hoffrage, \& Goldstein, 2008; Marewski \& Schooler,

This article was published Online First August 19, 2013.

M. Karl Healey and Michael J. Kahana, Department of Psychology, University of Pennsylvania.

This research was funded by National Institutes of Health Grant MH55687 and National Science Foundation Grant NSF1058886. We thank Lynn Lohnas, Ashwin Ramayya, and Joel Kuhn for helpful comments on this article; Jonathan Miller and Patrick Crutchley for assistance with designing and programming the experiment; and Kylie Hower, Joel Kuhn, and Elizabeth Crutchley for help with data collection.

Correspondence concerning this article should be addressed to M. Karl Healey or Michael J. Kahana, Department of Psychology, University of Pennsylvania, 3401 Walnut Street, Room 303, Philadelphia, PA 19104. E-mail: healeym@sas.upenn.edu or kahana@psych.upenn.edu
2011; Rieskamp \& Otto, 2006; Simon, 1956) and that the particular strategy a person selects can be highly sensitive to his or her individual goals and preferences (Carstensen, Isaacowitz, \& Charles, 1999).

Indeed, there has been much interest in the extent to which strategy use accounts for major findings in many areas including decision making (Payne, Bettman, \& Johnson, 1988), frequency judgment (Tversky \& Kahneman, 1973), students' study habits (Hartwig \& Dunlosky, 2012), working memory (Cusack, Lehmann, Veldsman, \& Mitchell, 2009; Ericsson \& Kintsch, 1995; Turley-Ames \& Whitfield, 2003), and long-term memory (Delaney, Spirgel, \& Toppino, 2012; Kahneman \& Wright, 1971; Paivio \& Yuille, 1969; Wright \& Kahneman, 1971), as well as for differences between individuals (Bailey, Dunlosky, \& Kane, 2008; Coyle, Read, Gaultney, \& Bjorklund, 1998) and groups of individuals such as younger and older adults (Dunlosky \& Hertzog, 1998, 2000; Mata, Schooler, \& Rieskamp, 2007). These views, though not generally framed as direct criticisms of using laboratory tasks to study particular cognitive systems (but see Hintzman, 2011), raise the question of whether performance on a laboratory task is so contaminated by individual differences in strategy as to be uninformative or even misleading about underlying cognitive processes.

To answer this question it is not enough to qualitatively describe individual differences in task behavior; rather, one must quantitatively test whether individuals deviate from the average in a way that substantially distorts the average pattern. Such quantitative tests are rare. Yet, given that data from laboratory paradigms provide the empirical foundation for much of psychological science, it is critical that the influence of idiosyncratic strategies be rigorously assessed. Here we develop an approach for distinguishing idiosyncratic strategies from core cognitive processes and 
apply it to a classic memory paradigm - free recall of word lists. We choose free recall both because it reveals a very detailed empirical pattern that has deeply influenced modern theories of memory (Davelaar, Goshen-Gottstein, Ashkenazi, Haarmann, \& Usher, 2005; Farrell, 2012; Polyn, Norman, \& Kahana, 2009) and because, of all the major memory tasks, it provides participants with the most opportunity to develop idiosyncratic encoding and retrieval strategies (Dalezman, 1976; Delaney \& Knowles, 2005; Delaney et al., 2012; Hintzman, 2011; Murdock \& Metcalfe, 1978; Sahakyan \& Delaney, 2003; Stoff \& Eagle, 1971).

\section{Free Recall and the Dynamics of Memory Search}

Many laboratory memory tasks require participants to retrieve very specific information, thereby imposing strong constraints on the scope of memory search. For example, serial recall forces participants to reconstruct the temporal order of events. Recognition provides a very strong external retrieval cue that constrains the scope of memory search, as does the paired-associates task. The great advantage of these tasks is that the strong constraints allow researchers to study specific memory processes while controlling other factors. Outside the laboratory, however, one must often search memory without strong external retrieval cues. If I ask you what you did last weekend, you are likely to search your memory for the various things you did, but the question itself does not provide any strong cues to guide you to a particular activity, nor does it constrain the order in which you recall your activities. Instead, you must take the vague cue of "what you did last weekend" and elaborate it to retrieve a specific activity. Once a first activity is retrieved associations between various activities are likely to govern their order of recall.

Many aspects of this natural memory search are captured by the free recall task, which allows participants to recall the studied items in any order. Therefore, the order of a sequence of recalls provides a window on memory search, allowing researchers to study search processes with precision and rigor. Free recall has helped illuminate important principles of memory search such as recency (recent events tend to be more memorable than distant events; Murdock, 1962), primacy (the first events in a sequence tend to be more memorable; Murdock, 1962), temporal contiguity (items that were experienced close together in time tend to be recalled contiguously; Kahana, 1996), and semantic proximity (items that are semantically related tend to be recalled together; Bousfield, Sedgewick, \& Cohen, 1954; Howard \& Kahana, 2002; Romney, Brewer, \& Batchelder, 1993).

We focus on four functions that provide a summary of the dynamics of memory search in free recall: the serial position curve (SPC), the probability of first recall (PFR) function, the lagconditional response probability (lag-CRP) function, and the semantic-conditional response probability (semantic-CRP) function. To illustrate the functions, we use data from the Penn Electrophysiology of Encoding and Retrieval Study (PEERS). The study is described in detail in the Method section, but briefly, participants studied multiple lists, each composed of 16 words, for immediate free recall.

The first function we consider is the classic SPC, which breaks down overall probability of recall by serial position at presentation. The SPC reveals the primacy and recency effects (see Figure 1A). In immediate free recall, recency dominates and the primacy effect is modest. The remaining functions we consider provide a detailed picture of the dynamics of memory search by measuring how participants initiate recall and how they transition among items after initiation.

Recall initiation can be examined by looking at which item participants tend to recall first and then computing a PFR function, an SPC based only on the very first item recalled (Hogan, 1975; Howard \& Kahana, 1999; Laming, 1999). As seen in Figure 1B, participants tend to initiate recall from the last serial position (Deese \& Kaufman, 1957).

After recall initiation, subsequent recalls are driven by associations between the just-recalled word and other words in the lexicon (Kahana, Howard, \& Polyn, 2008). Both preexisting semantic associations and newly formed episodic, or temporal, associations exert a powerful influence on recall transitions. In general, if a participant has just recalled item $i$ from the list, we can measure how $i$ 's associations with other items influence which item, $j$, is recalled next. To measure the influence of temporal associations, we can compute the probability that $i$ is followed by $j$ conditional on the distance, or lag, between $i$ and $j$ in the original list. For example, if $i=5$ and $j=6$, we would have a lag, $j-i$, of +1 . Lag-CRP functions give these probabilities for a variety of lags and are computed by dividing the number of times a transition of a given lag was actually made by the number of times it could have been made (Kahana, 1996). Note that lags that would lead outside the list boundaries are not considered possible (e.g., a +2 lag is impossible after recalling the 15 th item in a 16 -item list), nor are transitions to items that have already been recalled. Lag-CRP functions (see Figure 1C) reveal the contiguity effect: Transitions are most likely between temporally adjacent items and decrease in probability with increasing lag. In immediate free recall, the contiguity effect tends to be larger for the first few items recalled than for later output positions due to the strong recency effect (Davelaar et al., 2005; Sederberg, Howard, \& Kahana, 2008). Therefore we exclude the first two outputs from the lag-CRP analyses in this article. The lag-CRP also shows a forward asymmetry such that forward transitions are more likely than backward transitions for small absolute values of lag.

Lag-CRPs show how the probability of transitioning between two items is influenced by the proximity (i.e., lag) of those items along a temporal associative dimension, but semantic associations also exert a powerful influence on memory search (Bousfield, 1953; Romney et al., 1993). To measure the influence of semantic associations on transition probabilities, we can employ the same procedure used for temporal associations but replace temporal lag with a measure of semantic proximity. We use latent semantic analysis (LSA; Landauer \& Dumais, 1997) to measure semantic proximity. LSA allows one to measure the semantic relationship between two words as the cosine of the angle between the words' representations in a multidimensional model of semantic space. Using LSA values, we can compute the semantic similarity between item $i$ and all other items in the list. We can then bin pairs of items based on their semantic similarity and calculate the probability of making a transition from $i$ to $j$ based on the similarity bin of that pair. As seen in the resulting semantic-CRP function (see Figure 1D), there is a strong tendency to make transitions between semantically related words (Howard, Jing, Addis, \& Kahana, 2007; Howard \& Kahana, 2002; Sederberg, Miller, Howard, \& Kahana, 2010). 
A

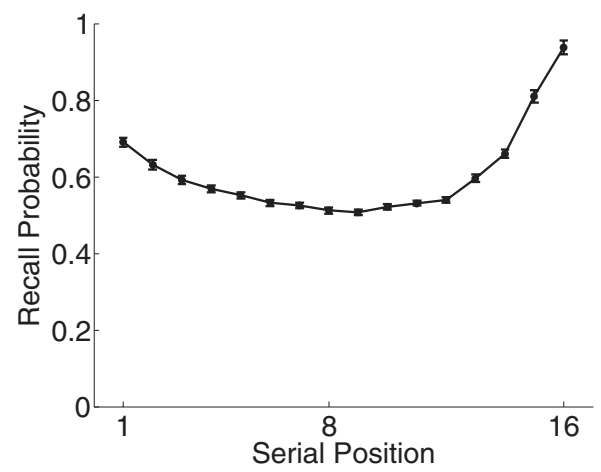

C

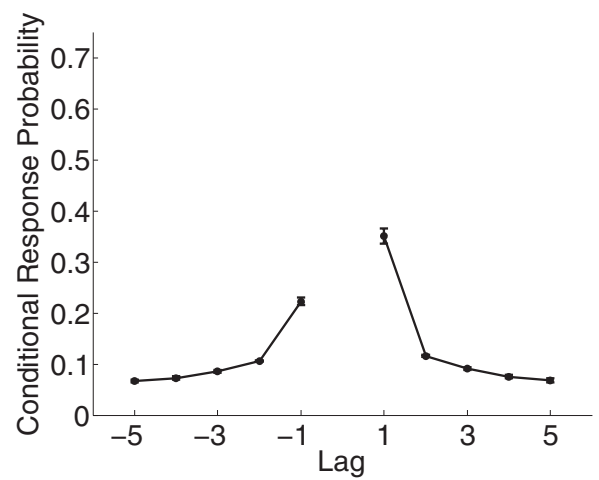

B

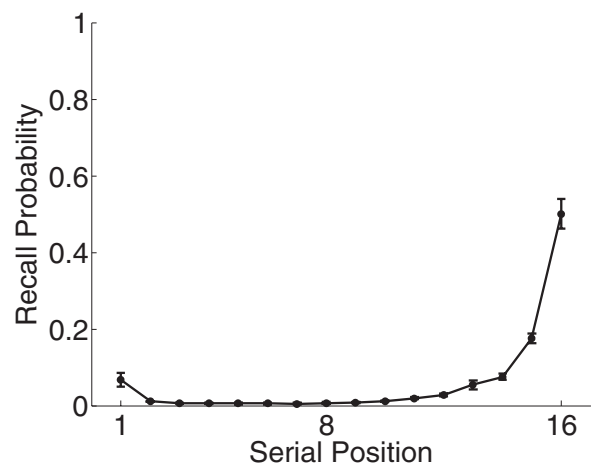

D

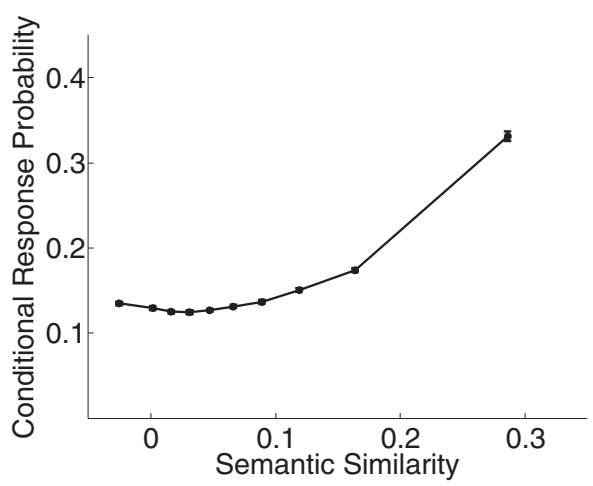

Figure 1. Data from the Penn Electrophysiology of Encoding and Retrieval Study: Serial position curve (A), probability first recall function (B), lag-conditional response probability function (C), and semantic-conditional response probability function (D). Error bars are 95\% within-subject confidence intervals (Loftus \& Masson, 1994).

These effects are quite robust. To our knowledge there is no published failure to find a temporal contiguity effect in a standard free recall task (but see McDaniel, Cahill, Bugg, \& Meadow, 2011, for a failure to find contiguity in recall of lists composed of orthographically distinct items), and the effect also emerges in a variety of other tasks such as high-confidence old/new recognition responses (Schwartz, Howard, Jing, \& Kahana, 2005) and intrusions in paired-associate recall (Davis, Geller, Rizzuto, \& Kahana, 2008). Robust semantic clustering effects in recall of categorized lists have been widely reported (Bousfield et al., 1954; Romney et al., 1993). Although the semantic proximity effect in uncategorized lists revealed by using LSA to construct semantic-CRPs has not been investigated as extensively, the effect is quite consistent across multiple independent data sets (Bridge, 2006; Howard \& Kahana, 1999; Kahana \& Howard, 2005; Kahana, Howard, Zaromb, \& Wingfield, 2002; Sederberg et al., 2006; Zaromb et al., 2006) as illustrated by the meta-analyses of Sederberg et al. (2010). Recency is somewhat more variable, being sensitive to the ratio of the length of the delay between successive items to the length of the delay between the final item and recall (Glanzer \& Cunitz, 1966; Postman \& Phillips, 1965), and other factors such as presentation modality (Murdock \& Walker, 1969). Primacy also varies, being closely tied to the frequency, recency, and spacing of rehearsals (Laming, 2006, 2008; Marshall \& Werder, 1972; Modigliani \& Hedges, 1987; Rundus, 1980; Tan \& Ward, 2000;
Ward, 2002). But the variables that influence primacy and recency are well understood, and many of their effects are predicted by several contemporary models of episodic memory (e.g., Farrell, 2012; Polyn et al., 2009).

Free recall data have played a major role in development of memory theory over the past 50 years. For example, the presence of recency in immediate free recall, and the absence of recency when there is a delay between the last item and recall (Glanzer \& Cunitz, 1966; Postman \& Phillips, 1965), is one of the strongest pieces of evidence supporting the distinction between immediate and secondary memory (Atkinson \& Shiffrin, 1968; Glanzer \& Cunitz, 1966; Waugh \& Norman, 1965). The temporal contiguity effect helped solidify the role that context has long played in episodic memory theories (Bower, 1972; Estes, 1955; McGeoch, 1932), and is now a benchmark effect that any model of episodic memory must account for (Davelaar et al., 2005; Farrell, 2012; Polyn et al., 2009).

\section{Principles of Memory or Artifacts of Strategy}

Despite the contributions of free recall, there remains some question as to whether the task actually captures fundamental principles of the memory system. Some scholars have expressed concern that free recall requires searching memory in a way that is alien to how memory is accessed outside the laboratory, which 
encourages use of idiosyncratic strategies. For example, Hintzman (2011) argued that participants in a free recall experiment are obligated to devise ad hoc mnemonic strategies and that individual differences in the type of strategy devised are likely to overlay and obscure more general memory processes:

\begin{abstract}
Allowing subjects to recall the words in any order they wish encourages them to explore various encoding and retrieval strategies. (p. 255)

But the overlay of study and retrieval strategies makes the task a grotesque, neither-fish-nor-fowl creature of the laboratorycorresponding to nothing people do in everyday life and too complex to be of much use for scientific analysis. (p. 255)
\end{abstract}

Others have made similar, if less sweeping, claims that various aspects of free recall performance are either artifacts of strategy use or modulated by strategy use (e.g., Dalezman, 1976; Delaney et al., 2012; Hasher, 1973; McDaniel \& Bugg, 2008).

Without doubt, participants will adopt strategies to help them perform free recall or any other laboratory task. The issue we wish to address here is whether variation across individuals in strategy use accounts for the shape of the average curves shown in Figure 1. Specifically, are the curves the result of averaging across subgroups of participants who are using different strategies, in which case they would tell us little about memory principles. Before describing how we will address this issue, it is important to clarify the distinction between strategies and principles of memory.

Performance on laboratory memory tasks is not a pure measure of memory. Rather, performance represents a complex interaction between characteristics of the participants, stimuli, the encoding conditions, and the retrieval conditions (Jenkins, 1979; Roediger, 2008). More generally, we can think of laboratory tasks as puzzles that require the participant to find the optimal way to use memory to meet the task requirements. For example, free recall and serial recall both rely on the same underlying memory system (Solway, Murdock, \& Kahana, 2012), but serial recall requires participants to place more weight on newly learned temporal associations and less weight on long-standing semantic associations.

A rough analogy can be made to chess playing. Chess is like a laboratory task, with the rules of the game placing constraints on how it can be played; the brain of the chess player is not preequipped with any "chess processes," just as there are no "free recall processes." Instead the player must take existing perception, memory, and executive processes and devise a strategy to deploy these processes in a way well-suited to the rules of the game. If we were to find patterns in how people play the game, for example, that they tend to plan only $x$ moves ahead, those effects could arise either from the particular strategies people adopt or from more fundamental principles of the cognitive systems those strategies deploy.

This view of task performance as an interaction between taskspecific strategy selection and domain-general memory processes is captured by several influential theories in cognition. The notion of "working with memory" (Moscovitch \& Winocur, 2002), for example, proposes that frontal executive processes must determine how best to deploy medial temporal lobe memory processes to meet the demands of the particular task. A similar view of task performance underlies the idea of the multiple-demand system (Duncan, 2010), which suggests that complex behavior depends on a network of frontal regions that break a task into smaller sub- components that can be processed by other cognitive systems such as memory and perception. Similarly, the idea of general-purpose cognitive systems being recruited in novel ways to address new tasks lies at the heart of production systems such as ACT-R (Anderson et al., 2004).

Characteristics of both the task and the participants will influence the balance between the strategy and memory process components of free recall performance. A variety of task characteristics such as presentation rate, presentation modality, and list length have been shown to influence the shape of free recall functions. More generally, if we consider task parameters such as list length to be a continuum, there are likely to be points along this continuum where the optimal strategy shifts (Grenfell-Essam \& Ward, 2012). Indeed, GrenfellEssam and Ward (2012) have shown that for short list lengths many participants tend to initiate recall from the beginning of the list rather than from the end of the list, as seen with the longer lists we use here (see Figure 1). Therefore, studies designed to capture the memory component should avoid crossover points at which the strategy component is likely to be highly influential.

An important characteristic of participants that will affect the balance between the strategy and memory process components is the amount of practice they have with the task. When first introduced to a new task, strategy differences are likely to be prominent, as participants explore different strategies in an attempt to find an optimal one. Indeed, the findings that recall initiation patterns change with practice (Dallett, 1963; Goodwin, 1976; Hasher, 1973; Huang, 1986) and that memory task performance tends to improve with practice (i.e., the "learning to learn" effect; Postman, 1969) may stem partly from participants optimizing strategies to meet the needs of the tasks (we revisit this suggestion in the Discussion and make an important caveat). Therefore, we examine the performance of participants with extensive experience with the free recall task.

However, using well-practiced participants is not enough to ensure that the effects shown in Figure 1 reflect general memory processes. It is possible that even after many trials of practice, differences in strategy use between individuals may remain and that these differences may be obscured when looking at averaged data. In this article, we develop an analytical framework for detecting such strategy differences.

\section{Establishing Consistency Across Individuals}

In developing this analytical framework, we start by identifying differing predictions of the strategy-difference and memoryprocess accounts of free recall effects. One guiding principle is that if the functions in Figure 1 reflect fundamental principles of the memory system, then they should be universal in the sense that every cognitively intact individual should exhibit a similar function, just as every healthy individual shows markers of fundamental physiological processes. There will, of course, be individual differences, but they should be quantitative rather than qualitative. By contrast, a strategy account predicts that the shape of a participant's function will depend on the strategy they use.

Specifically, if any of the effects described above are the result of averaging across subgroups of participants who are using different strategies, each subgroup should correspond to a distinct and identifiable cluster in the data. Assume, for example, that the average curves actually disguise a "primacy" subgroup that adopts 
the strategy of initiating recall with items from early serial positions and a "recency" subgroup that adopts a strategy of initiating with end-of-list items, similar to the two initiation patterns identified by Grenfell-Essam and Ward (2012). We should be able to identify these two clusters by examining the PFR functions of individual participants. One could imagine many other ways in which strategy use could influence PFR functions. And similar strategy-based accounts could be made for the contiguity and proximity effects (e.g., there may be a cluster of participants that shows strong contiguity and another that shows weak contiguity). We develop a generalizable strategy detection procedure that is based on identifying clusters in the data but does not require specifying the particular strategies, the number of clusters, or how strategies change the shape of the functions.

In addition to the cluster detection procedure, we use the strategy-free context maintenance and retrieval (CMR) model of free recall (Polyn et al., 2009) to test the strategy account. Unsworth, Brewer, and Spillers (2011) pointed out that although several existing models fit a wide range of free recall effects, they do so at the level of average data and do not model individual differences in strategy use. This view implies that existing models would be unable to fit data at the individual level without being modified to incorporate a strategy component. However, whether models need to include strategy components to account for individual data has not been extensively investigated. The issue actually suggests a strong test of the claim that free recall effects cannot be explained apart from reference to strategy: If a model without a strategy component can fit individual difference data, it constitutes a sufficiency proof - conclusively showing that reference to strategy is unnecessary to account for the existing data.

\section{Method}

\section{Participants}

The data reported here are from the Penn Electrophysiology of Encoding and Retrieval Study (PEERS). PEERS aims to assemble a large database on the electrophysiological correlates of memory encoding and retrieval. The present analyses are based on the 126 young adult (age 17-30) participants who had completed Experiment 1 of PEERS as of December 2012. Participants were recruited through a two-stage process. First, we recruited righthanded native English speakers for a single session to introduce participants to electroencephalogram recordings (electroencephalogram data are not reported here) and the free recall task. Participants who completed this introductory session were invited to enroll in the full study, on the condition that they did not make an excess of eye movements during item presentation epochs of the experiment and their probability of recall was less than .8. Approximately half the subjects recruited for the preliminary session agreed to participate in the multisession study. Participants were consented according the University of Pennsylvania's institutional review board protocol and were compensated for their participation.

\section{PEERS Experiment 1}

Participants performed a free recall experiment consisting of one practice session and six subsequent experimental sessions.
Each session consisted of 16 lists of 16 words presented one at a time on a computer screen. Each study list was followed by an immediate free recall test, and each session ended with a recognition test. Half the sessions were randomly chosen to include a final free recall test before recognition, in which participants recalled words from any of the lists from the session.

Words were presented concurrently either with a task cue, indicating the judgment that the participant should make for that word, or with no encoding task. The two encoding tasks were a size judgment ("Will this item fit into a shoebox?") and an animacy judgment ("Does this word refer to something living or not living?"), and the current task was indicated by the color and typeface of the presented item. Based on the results of a prior norming study, only words that were clear in meaning and that could be reliably judged in the size and animacy encoding tasks were included in the pool. There were three conditions: no-task lists (participants did not have to perform judgments with the presented items), single-task lists (all items were presented with the same task), and task-shift lists (items were presented with either task). The first two lists were task-shift lists, and each list started with a different task. The next 14 lists contained four no-task lists, six single-task lists (three of each task), and four task-shift lists. List and task order were counterbalanced across sessions and participants.

Each word was drawn from a pool of 1,638 words. Lists were constructed such that varying degrees of semantic relatedness occurred at both adjacent and distant serial positions. Semantic relatedness was determined using the word association space model described by Steyvers, Shiffrin, and Nelson (2004). Word association space similarity values were used to group words into four similarity bins (high similarity, $\cos \theta$ between words $>.7$; medium-high similarity, $.4<\cos \theta<.7$; medium-low similarity, $.14<\cos \theta<0.4$; low similarity, $\cos \theta<.14$ ). Two pairs of items from each of the four groups were arranged such that one pair occurred at adjacent serial positions and the other pair was separated by at least two other items.

For each list, there was a 1,500-ms delay before the first word appeared on the screen. Each item was on the screen for 3,000 ms, followed by jittered 800 - to 1,200 -ms interstimulus interval (uniform distribution). If the word was associated with a task, participants indicated their response via a keypress. After the last item in the list, there was a 1,200- to 1,400-ms jittered delay, after which a tone sounded, a row of asterisks appeared, and the participant was given $75 \mathrm{~s}$ to attempt to recall any of the just-presented items.

If a session was selected for final free recall, following the immediate free recall test from the last list, participants were shown an instruction screen for final free recall, telling them to recall all the items from the preceding lists. After a 5-s delay, a tone sounded and a row of asterisks appeared. Participants had 5 min to recall any item from the preceding lists.

After either final free recall or the last list's immediate recall test was a recognition test, indicated by an instruction screen. Target to lure ratio was variable by session, where targets made up $80 \%, 75 \%$, $62.5 \%$, or $50 \%$ of the total items. Participants were told to respond verbally by saying "pess" for old items and "po" for new items and to confirm their response by pressing the space bar. This was done so that both response types would initiate with the same stop consonant (or plosive) so as to assist in automated detection of word onset times. Following the old/new judgment, participants made a confidence 
rating on a scale of 1 to 5 , with 5 being the most confident. Recognition was self-paced, though participants were encouraged to respond as quickly as possible without sacrificing accuracy. Participants were given feedback on accuracy and reaction time.

The first session was identical to the experimental sessions except that this session always had final free recall. To make it easier for participants to adjust to the encoding task, lists were not presented randomly in the first session. Rather, Lists 1-4 were no-task lists, Lists 5-8 were single-task lists of one randomly selected judgment type, Lists 9-12 were single-task lists using the other judgment, and Lists 13-16 were task-shift lists. The number of item presentations for each judgment type were counterbalanced across lists.

\section{Results}

\section{Overview of Data}

In determining whether the free recall effects are consistent across participants, it is useful to examine the free recall functions for individual participants. As described in the Method section, PEERS includes an encoding task manipulation; task trials require participants to make either a size or an animacy judgment about each word as it is presented, whereas no-task trials have no encoding task. The requirement to perform a specific task during encoding may influence a participant's choice of strategy. Therefore we analyze task and no-task conditions separately.

Figures 2-5 show SPC, PFR, lag-CRP, and semantic-CRP functions, respectively. In the figures, a separate panel is devoted to each participant. Each panel displays four curves. The solid curves show no-task lists, and the dashed curves show task lists. The black curves show the participant's data, and the gray curves show the fit of the context maintenance and retrieval (CMR) model to their data (see below for details on the model and how it was fit to the data). The ordering of participants is consistent across figures, so that a given participant appears in the same panel in each figure. ${ }^{1}$ We present detailed quantitative analyses below, but the data are sufficiently clear to begin with a visual inspection and descriptive overview.

Figure 2 shows each participant's SPC. Although there are clearly individual differences, every participant's function resembles a classic SPC. If we define recency as a higher average recall probability for the final six serial positions than for Positions 5-10 (mid-list positions), then on both no-task and task lists $90 \%$ of participants show recency. If we define primacy as higher average probabilities for Items 1-4 than for mid-list positions, then for both no-task and task lists $93 \%$ of participants show primacy.

Individual PFR functions are shown in Figure 3. The PFR peaks at the final item for $64 \%$ of participants on no-task lists and for $79 \%$ participants on task lists. The PFR is uniformly near 0 for mid-list positions: Across all participants the average initiation probability for Items $5-10$ is less than $1 \%$ for both no-task and task lists. No participants show an average initiation probability of more than $5 \%$ for mid-list items on no-task lists (one participant does so on task lists). Individual PFRs do, however, vary somewhat from the average. First, on no-task lists, $27 \%$ of participants (16\% on task lists) are more likely to initiate recall from an item one to three positions back from the final item than from the final item itself. Second, $44 \%$ ( $27 \%$ on task lists) of participants initiate recall from the beginning of the list on more than $5 \%$ of trials, producing an uptick in the PFR at the first item. Overall, the PFR average function is a reasonable description of the modal participant. A substantial number of participants do depart from this modal function, but they do not greatly distort the average (with the possible exception of a small uptick for the first item, which is visible in the average function).

The individual participant lag-CRP functions (see Figure 4) show remarkable consistency. If we define a contiguity effect as the CRP for Lag +1 being greater than the CRP for Lag +2 and the CRP for Lag -1 being greater than the CRP for Lag $-2,96 \%$ of participants show a contiguity effect on no-task lists, and $100 \%$ show contiguity on task lists. As a more stringent test, we created a 6-point lag-CRP function composed of $\mathrm{Lag}+1$, the averages of Lags +2 and +3 , the averages of Lags +3 and +5 , and the corresponding points for negative lags. These functions decline monotonically with increasing absolute value of lag for $98 \%$ of participants on no-task lists and $100 \%$ of participants on task lists. The asymmetry effect is also highly consistent; $95 \%$ of participants show higher recall probabilities for Lag +1 than for Lag -1 on no-task lists (96\% of participants show asymmetry on task lists). There are, of course, individual differences, with some participants showing large asymmetries and others showing very small asymmetries. However, these differences are quantitative rather than qualitative, as would be expected if all participants' asymmetry effects were drawn from a normal distribution centered above 0 with a left tail close to 0 .

The semantic proximity effect also shows striking consistency across participants (see Figure 5). For all participants, conditional recall probability peaks at the highest similarity bin and decreases as semantic similarity decreases. No participants show a flat or negatively sloped function.

Even though it is not our primary focus, it is useful to compare the no-task and task conditions. On average, SPC curves tend to be lower for the task lists and primacy is more pronounced for no-tasks lists. Temporal contiguity also tends to be lower on the task lists. These differences suggest that the encoding task influences how participants approach the task; however, it is not clear whether this reflects differences in the strategy component or the memory component.

Figures 2-5 reveal an impressive level of consistency across participants and strongly suggest that a common set of memory processes underlie the functions. Yet, few individuals show a pattern that is identical to the average. Of course, visual inspection does not tell us whether we can reject the null hypothesis that a given participant's curve actually deviates significantly from the average. Therefore, we turn to a series of analyses that provide a more rigorous test.

\section{Detailed Analyses}

We develop a two-step procedure for detecting strategy subgroups. The first step is a cluster detection algorithm and is suited to situations in which participants fall into clear subgroups. The

\footnotetext{
${ }^{1}$ The ordering of participants (down rows first, then across columns) is based on membership in the subgroups identified in the PFR curve (see the Detailed Analyses section). Specifically, participants belonging to the first cluster in Figure 9 are plotted first, followed by participants belonging to the second cluster, and so on.
} 


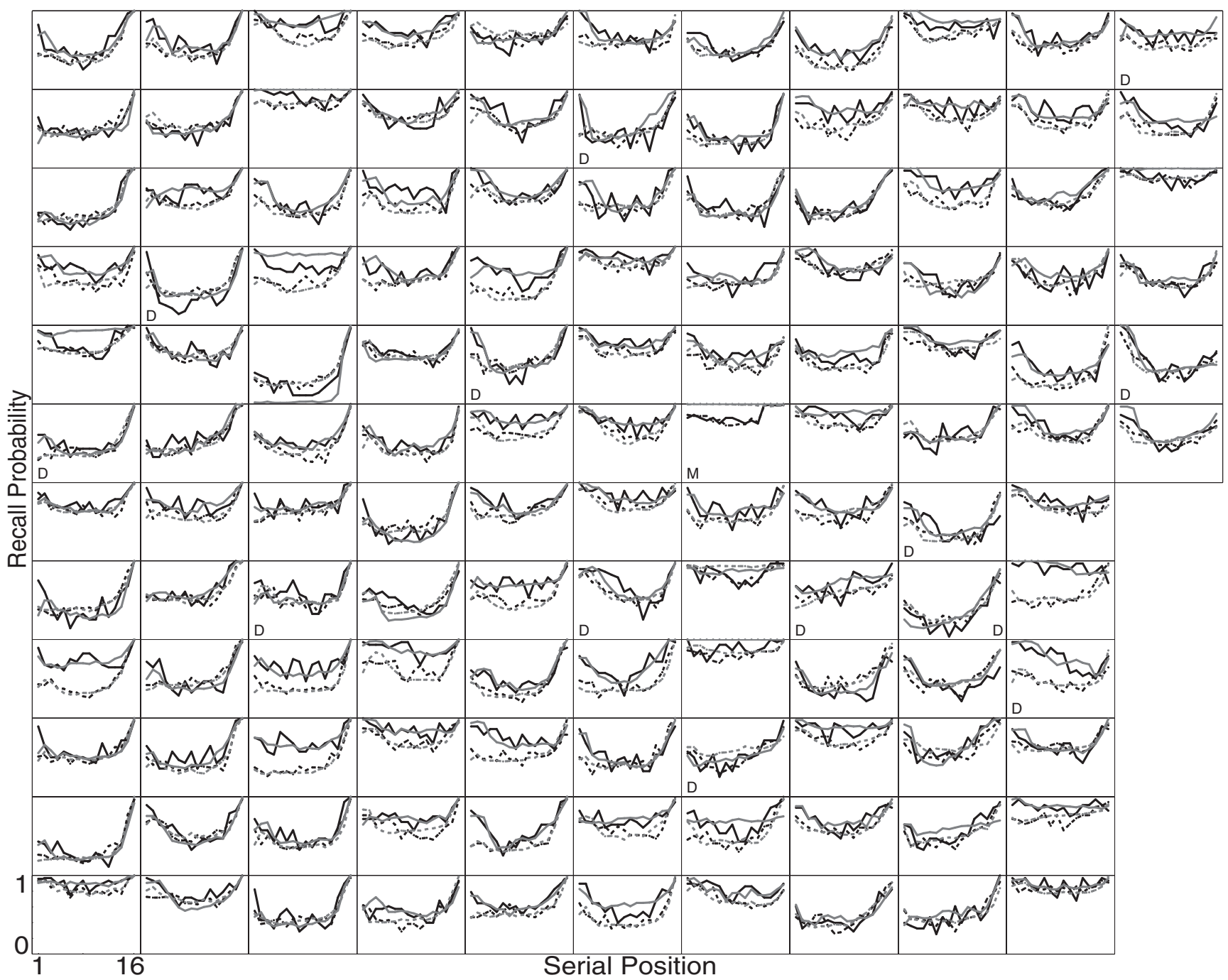

Figure 2. Individual participants' serial position curves. Each panel shows the data from one participant. Participants are displayed in the same order in each of Figures 2-5. Solid lines show the no-task lists; dashed lines show the task lists. Black lines are each participant's actual data. Gray lines are fits of the context maintenance and retrieval (CMR) model to the participant's data. A $D$ in a participant's panel indicates that the individual differed significantly from the average function on at least one list type; an $M$ indicates that CMR failed to provide a good fit to the data for at least one list type. The electronic version of the article includes high-resolution figures that allow for zooming in on individual plots.

second step is to test each individual's deviance from the average and is intended for cases in which the fist step fails to detect large clusters.

We will use the PFR curve to illustrate the first step in our approach. We created a simulated "strategy" data set composed of two distinct groups of simulated participants; the first group shows a strong recency effect but no primacy (see Figure 6A), and the second shows strong primacy but no recency (Figure 6B). By combining these two groups in a 75:25 ratio, we get an average PFR function that resembles real data (Figure 6C). We can test the null hypothesis that the data set contains a single cluster by calculating the mean-squared deviation of observations from the overall mean and then determining how much we can reduce the this deviation by assigning the data to $k$ clusters and getting the deviation of each observation from its cluster mean rather than from the overall mean. The challenge for cluster detection algorithms is that mean-squared error is a monotonically decreasing function of $k$, and approaches 0 as $k$ approaches the number of observations (i.e., each data point is its own cluster).

There are many existing cluster detection algorithms (e.g., Garrido, Abad, \& Ponsoda, 2012; Pelleg \& Moore, 2000; Pham, Dimov, \& Nguyen, 2005; Sugar \& James, 2003); however, most deal with this overfitting problem by relying on arbitrary rules of thumb (e.g., looking for an "elbow" or inflection point in a plot of error against values of $k$ ) or on generic corrections for overfitting (e.g., the Akaike and Bayesian information criteria). Instead we use a principled method (Sugar \& James, 2003) based on the intuition that the correct number of clusters should provide a 




Figure 3. Individual participants' probability of first recall functions. Each panel shows the data from one participant. Participants are displayed in the same order in each of Figures 2-5. Solid lines show the no-task lists; dashed lines show the task lists. Black lines are each participant's actual data. Gray lines are fits of the context maintenance and retrieval (CMR) model to the participant's data. An $M$ in a participant's panel indicates that CMR failed to provide a good fit to the data for at least one list type. The electronic version of the article includes high-resolution figures that allow for zooming in on individual plots.

greater decrease in error than any other value of $k$. The algorithm starts by assigning the data to $k$ clusters (here we use $k$-means for cluster assignment) for a range of values of $k$ (here we use $1-4){ }^{2}$ Then for each value of $k$ it computes, $d_{k}$, the average Mahalanobis distance of data points from their cluster centers divided by the number of dimensions in the data:

$$
d_{k}=\frac{1}{p} \frac{\sum_{i=1}^{n}\left(\mathbf{X}_{i}-\mathbf{c}_{x_{i}}\right)^{T} S^{-1}\left(\mathbf{X}_{i}-\mathbf{c}_{x_{i}}\right)}{n},
$$

where $p$ is the number of dimensions in the data, $X_{i}$ is the $i$ th participant's data as a column vector, $S$ is the covariance matrix, $n$ is the number of subjects, and $C_{x_{i}}$ is the center of the $i$ th participant's cluster. The next step is to raise $d_{k}$ to a small negative power, $-Y$, so that it is an increasing function of $k$ with a fairly shallow slope, which reduces differences between adjacent values of $k$. The final step is to calculate the reduction in error, or "jumps," created by moving from $k-1$ to $k$ for each value of $k$. Specifically,

$$
J_{k}=d_{k}^{-Y}-d_{k-1}^{-Y} \text {. }
$$

\footnotetext{
${ }^{2}$ We found that using values of $k>4$ produced clusters that contained only a few participants and that cluster membership was not stable across runs of the algorithm. The Monte Carlo procedure we introduce in the next section for detecting deviation of individuals from the average curves is better suited to detecting very small groups of participants that depart from the average curve.
} 


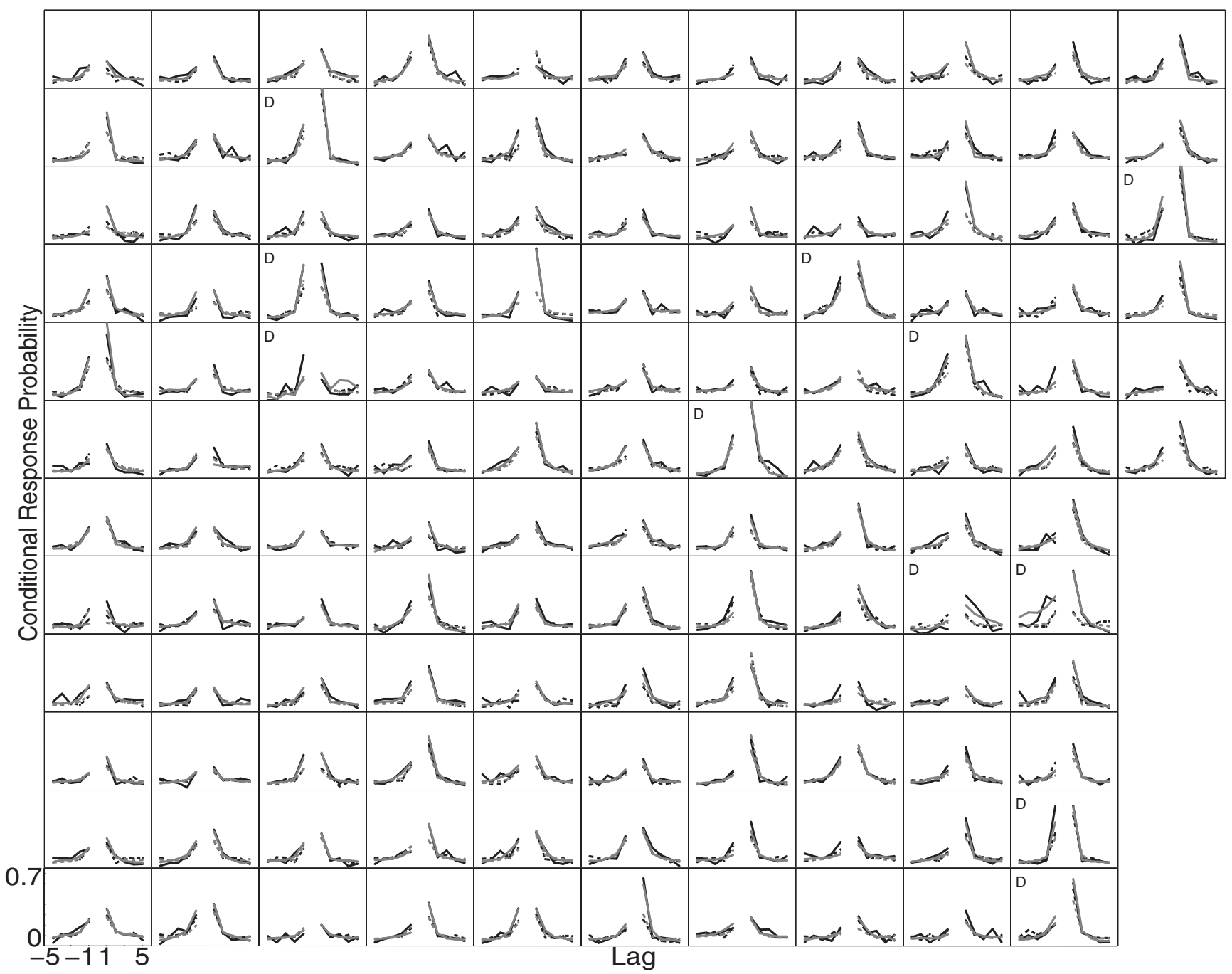

Figure 4. Individual participants' lag-conditional response probability functions. Each panel shows the data from one participant. Participants are displayed in the same order in each of Figures 2-5. Solid lines show the no-task lists; dashed lines show the task lists. Black lines are each participant's actual data. Gray lines are fits of the context maintenance and retrieval (CMR) model to the participant's data. A $D$ in a participant's panel indicates that the individual differed significantly from the average function on at least one list type; an $M$ indicates that CMR failed to provide a good fit to the data for at least one list type. The electronic version of the article includes high-resolution figures that allow for zooming in on individual plots.

Note that when $k=1, d_{k-1}$ is taken to be 0 so that a jump value can be calculated for one cluster. The largest jump should occur for the true value of $k$, which can easily be seen in a plot of $J_{k}$, against $k$. Sugar and James (2003) have shown that this method is highly effective at recovering the true value of $k$ in many simulated and real data sets, both with normal and nonnormal underlying distributions. We ran the algorithm for values of $k$ between 1 and 4 and took the $k$ with the largest jump to be the appropriate number of clusters. Noise in the data can blur the boundaries between true clusters, making them hard to detect. We overcame this challenge by performing the above-described fitting procedure on 5,000 bootstrap samples; the reported jump values are the averages over all samples. Bootstrapping also allows us to create confidence intervals around the values of $J_{k}$, which provides an natural test for the presence of significant clustering: If the data contain $k$ true clusters, $J_{k}$ should fall outside the $95 \%$ confidence interval for $J_{1}$.

We applied this procedure to both the simulated strategy data set and a no-strategy control set that had the same means as the strategy set but contained a single cluster. Figure 7 shows that the cluster detection procedure correctly determined that the simulated strategy data contain clusters, whereas the no-strategy data do not. To establish the effectiveness of this cluster detection procedure, we tested its ability to detect clusters in simulated data with ratios of recency to primacy simulated participants of 55:45, 65:35, $75: 25$, and 85:15. For each ratio we generated 1,000 samples of 126 simulated participants; the algorithm correctly identified all of these data sets as having more than one cluster, and tended to overestimate the actual value of $k$. That is, the procedure is highly 


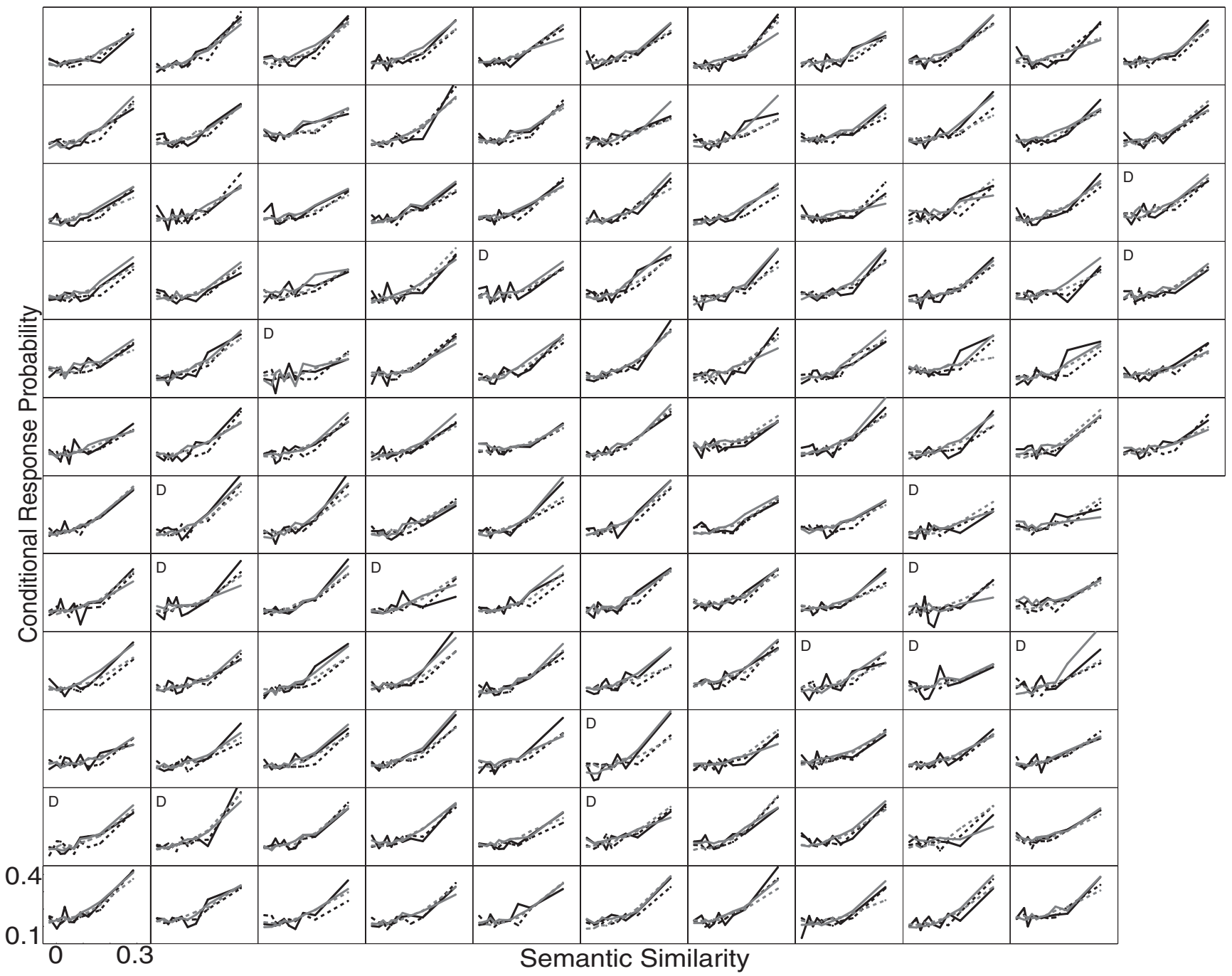

Figure 5. Individual participants' semantic-conditional probability functions. Each panel shows the data from one participant. Participants are displayed in the same order in each of Figures 2-5. Solid lines show the no-task lists; dashed lines show the task lists. Black lines are each participant's actual data. Gray lines are fits of the context maintenance and retrieval (CMR) model to the participant's data. A $D$ in a participant's panel indicates that the individual differed significantly from the average function on at least one list type; an $M$ indicates that CMR failed to provide a good fit to the data for at least one list type. The electronic version of the article includes high-resolution figures that allow for zooming in on individual plots.

accurate at detecting the presence of clusters composed of as little as $15 \%$ of the sample. The tendency to overestimate the value of $k$ makes it a conservative test of the claim that $k=1$. We explored a variety of other clustering algorithms such as Monte Carlo methods that compare multivariate distributions implied by $k$ clusters with the actual data, and found that these methods tended to underestimate the true value of $k$.

We applied the same analysis to the free recall curves of the actual participants. For the actual SPC, lag-CRP, and semanticCRP curves, the results in Figure 8 closely resemble the pattern shown by the no-strategy simulations. For each of these curves, we were unable to reject the null hypothesis that the data contained a single cluster. By contrast, the PFR curves showed evidence of clustering: The null of $k=1$ was rejected for both the no-task and task lists, and the jump functions peaked at $k=4$ and $k=3$ for each list type. To understand the differences in initiation patterns between the clusters, we used $k$-means clustering to assign each participant to one of four clusters and plot the average curves for each cluster in Figure 9.

The first two clusters show the same basic shape as the average PFR curve, and likely represent an artificial division of a continuous distribution at an arbitrary point. That is, both clusters show an "end-first" initiation pattern and simply differ quantitatively. The next two clusters, however, show qualitative deviation from the average curve. The third cluster represents participants who follow a "beginning-first" initiation pattern. This subgroup of participants resembles the initiation pattern found by GrenfellEssam and Ward (2012) with short lists. It appears that even with 
A

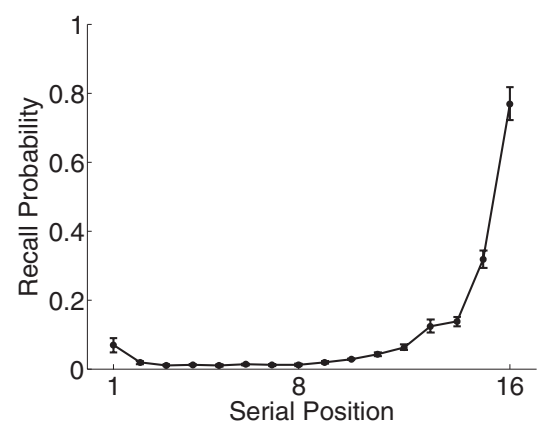

B

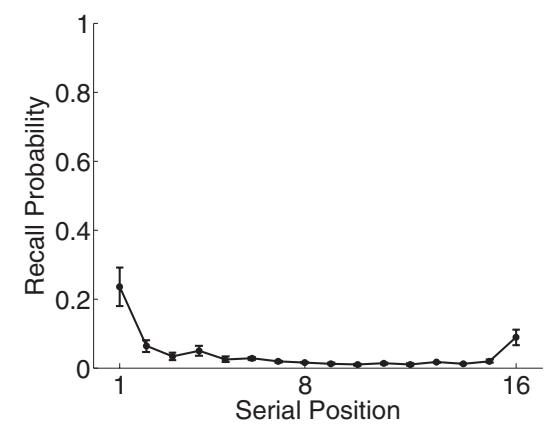

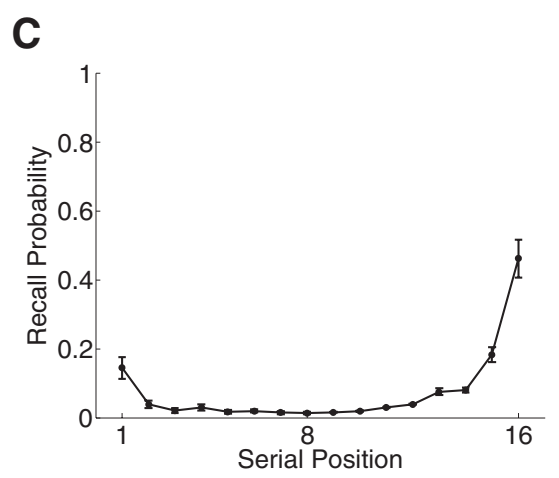

Figure 6. Probability of first recall curves from simulated participants: Data from 95 simulated participants, all showing recency but no primacy (A); data from 31 simulated participants, all showing primacy but no recency (B); and the average function from all 126 simulated participants, showing strong recency and weaker primacy (C). Error bars are 95\% within-subject confidence intervals (Loftus \& Masson, 1994).

long lists, a substantial number of participants retain the beginning-first pattern (note, however, that the number of participants showing this pattern is substantially smaller for task lists than no-task lists, suggesting an influence of encoding task on initiation pattern). The last cluster represents participants who tend to initiate at some point near, but not exactly at, the end of the list. This may reflect chunking (Farrell, 2012) in which participants encode trains of three to four successive items into a chunk and recall in forward order within chunks. Thus, the PFR function exhibits evidence of qualitative variation that may reflect individual differences in strategy. We stress, however, that these qualitative variations do not substantially distort the average function, which provides a very good description of the modal participant. It is not the case that the average PFR gets its shape from averaging across subgroups that do not resemble the average. Rather, it is the case that the average disguises two subgroups while accurately describing the largest group.

To provide a visual check on the claim that the SPC, lag-CRP, and semantic-CRP curves do not show clustering, we can use $k$-means to force the data into clusters and plot the cluster means as we did for PFR. In the absence of true clusters (i.e., if the data are drawn from a single distribution), we would expect $k$-means to simply divide the distribution into quantiles, which should be apparent as systematic variation of the curves across clusters (as opposed to the qualitatively different curves seen across clusters for PFR). For the SPC curve, such variation should appear as a lowering of the curve; for the contiguity curves, we would expect some clusters to have steeper contiguity effects than others but for all to show the same functional form (i.e., CRP changes nonlinearly with both temporal and semantic proximity; thus we would not expect a simple raising and lowering of the curves, but a change in slope). As can be seen in Figures 10-12, this is exactly the pattern we observe; each cluster shows the same basic shape as the average curve but with the level or slope differing systematically.

The foregoing clustering analyses confirm what is obvious from inspecting individual participants' data: That for the SPC, lagCRP, and semantic-CRP curves, the average effects are not due to substantial subgroups of participants performing the task in qualitatively different ways. The PFR curve provides an accurate description of the modal participant who follows an end-first recall initiation pattern, but disguises two subgroups that adopt a beginning-first and a clustering initiation pattern, respectively. The clustering analyses, however, do not tell us whether a particular individual is well described by the average. That is, even though coherent groups of participants do not deviate from the average pattern, individual participants may. This is an important point, as under the assumption that free recall is capturing something fundamental about the memory system, we would expect the functions to capture the performance of every healthy adult, just as we expect an anatomy textbook to qualitatively capture the anatomy of any healthy individual.

How do we test whether an individual's function is well described by the average function? Using SPC as an example, 
A

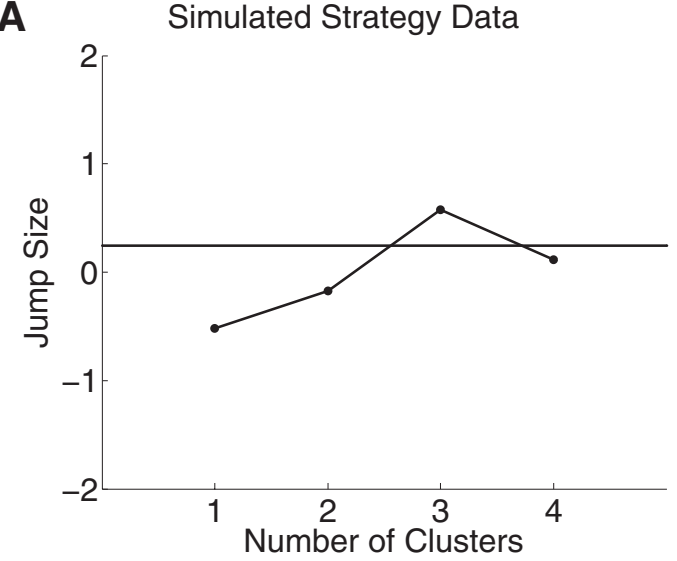

B

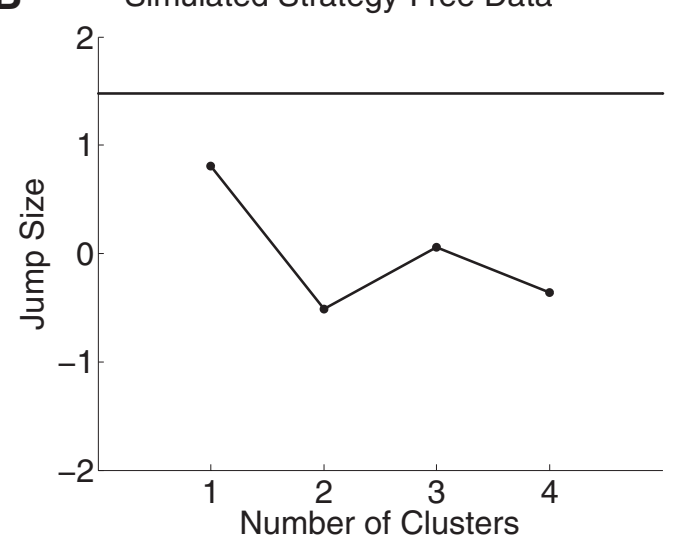

Figure 7. Results of cluster detection analyses for simulated participants: The results of the procedure applied to a sample composed of 31 simulated primacy participants and 95 simulated recency participants (A), and the results of the procedure applied to a sample composed of 126 participants all drawn from a distribution that shows both primacy and recency (B). Jump values were normalized across values of $k$ (i.e., the number of clusters) to place each plot on a common scale. The horizontal lines show the upper bound of a $95 \%$ confidence interval on the jump value for $k=$ 1 ; if the jump value for any $k$ exceeds this bound, we can reject the hypothesis that the data contain a single cluster, with the peak of the function indicating the appropriate number of clusters.

one could examine each serial position and determine whether the participant's score for that position deviated from the average. However, this approach ignores the fact that performance across serial positions is correlated. For example, it might not be unusual for a participant to be 1 standard deviation below the average at all serial positions, but it would be unusual for someone to be 3 standard deviations above the mean on the first few serial positions and 1 standard deviation below average for all other positions. That is, we want to know whether a participant's function taken as a whole is unusual.

Therefore, we started with the null hypothesis that each participant's function for a particular effect is the result of a random draw from a multivariate distribution described by the means and covariance matrix for that effect (e.g., the covariance of the Subjects $\times$ Serial Position matrix). For each participant we followed several steps designed to test whether this null hypothesis could be rejected; rejection of the null would suggest that the participant's data were not drawn from the average distribution. First, we computed the covariance matrix using the data from all participants except the one currently being considered (so that participants did not bias the results in their favor) and used the matrix to generate 100,000 simulated serial position curves with the same means, distributions, and covariance structure as the actual data. Next, for each simulated function we calculated its Mahalanobis distance from the rest of the simulated functions, providing us with a distribution of distances for functions actually drawn from the same underlying distribution. Finally, to determine whether the participant in question was also likely to be drawn from that distribution, we calculated the Mahalanobis distance of the participant's function from the simulated functions. If the participant's Mahalanobis distance was greater than $95 \%$ of the simulated Mahalanobis distances, that participant was designated as deviant. ${ }^{3}$

The same procedure was repeated for the SPC, lag-CRP, and semantic-CRP (we do not consider the PFR, as we have already established that subgroups deviate from the average). To avoid giving too much weight to noise in the individual curves, we averaged across certain data points. Specifically, we created reduced curves consisting of the following data points for each function: For SPC, each of Positions 1, 2, 3, and 4; the average of Positions 5-8; the average of Positions 9-12; and each of Positions 13, 14, 15, and 16; for lag-CRP, Lag 1, the average of Lags $2-3$, the average of Lags $4-5$, and the corresponding negative lags; for semantic-CRP, each of the individual bins. Participants who were designated as deviant are marked by the letter $D$ in their panel in Figures 2, 4, and 5.

For SPC, lag-CRP, and semantic-CRP, only 4\%, 4.8\%, and $7.14 \%$ of cases, respectively $(6.3 \%, 4.8 \%$, and $5.56 \%$ on task lists), were classified as deviant. Visual inspection of these cases suggests that they do not differ qualitatively from the average functions (see the marked cases in Figures 2 and 5); rather, deviant participants show the same qualitative pattern as the nondeviant subjects, but are near the tails of the nondeviant distribution (e.g., relatively steep or relatively shallow contiguity effects). Because of this qualitative similarity and the fact the these deviance rates are very near or below the $5 \%$ false detection rate for the procedure, we assume they are false alarms and do not consider them further.

To determine whether a participant's distance from the average of one function predicts his or her distance from the other functions, we correlated participants' Mahalanobis distances for each of the four functions. Mahalanobis distances are expected to be highly skewed; therefore, we used the nonparametric Spearman's $\rho$ rather than Pearson's $r$. The results are displayed in Table 1. In general, a participant's distance from the average of one function was not strongly correlated with his or her distance from the average of the other functions. However, distance from the lag-

\footnotetext{
${ }^{3}$ When the data matrix is multivariate normal, squared Mahalanobis distances are distributed as chi-square with degrees freedom equal to the dimensionality of the data. Using this fact to test whether participants significantly deviated from average provided results very similar to those provided by the simulation method described in the text.
} 
A

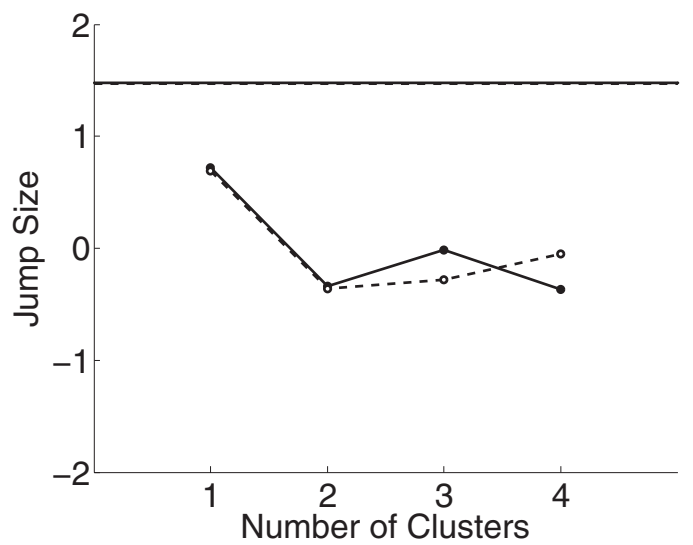

C

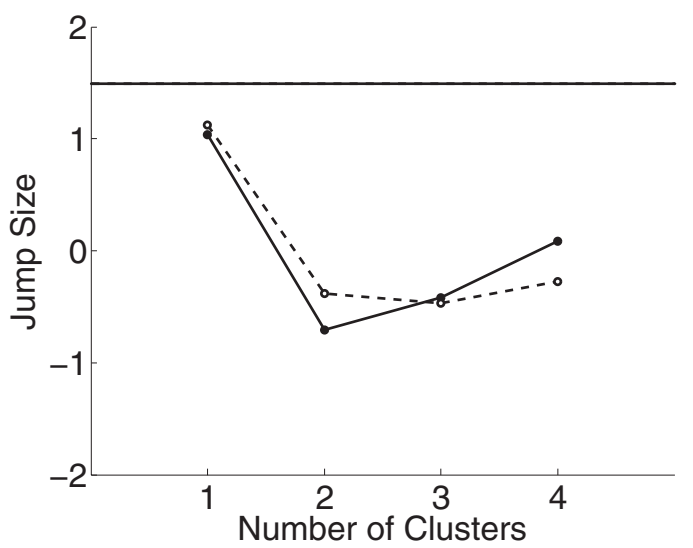

B

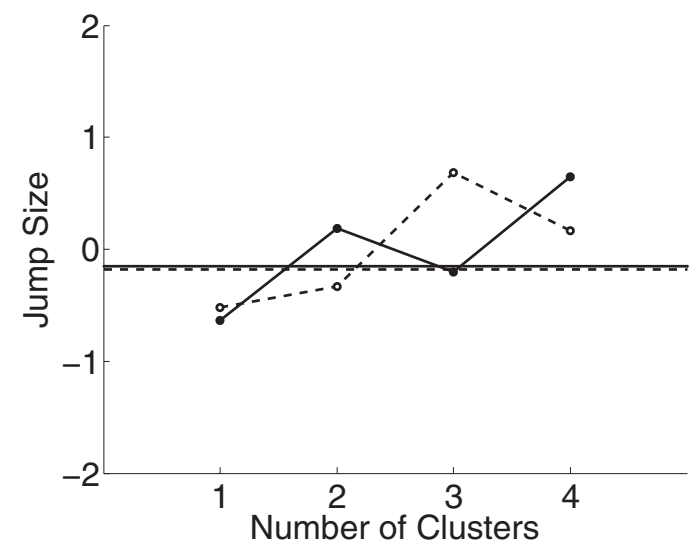

D

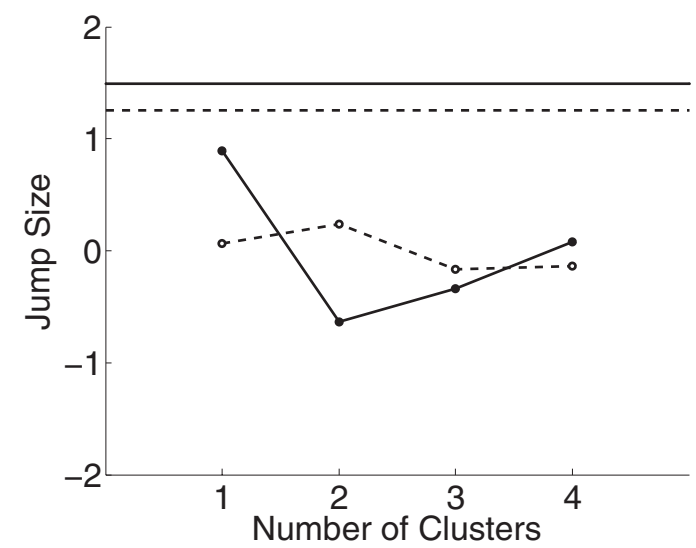

Figure 8. Results of cluster detection analyses for actual participants: Serial position curve (A), probability first recall function (B), lag-conditional response probability function (C), and semantic-conditional response probability function (D). Solid lines are no-task lists; dashed lines are task lists. Jump values were normalized across values of $k$ (i.e., the number of clusters) to place each plot on a common scale. The horizontal lines show the upper bound of a $95 \%$ confidence interval on the jump value for $k=1$; if the jump value for any $k$ exceeds this bound, we can reject the hypothesis that the data contain a single cluster, with the peak of the function indicating the appropriate number of clusters.

CRP was moderately but significantly correlated with distance from both semantic-CRP and PFR.

\section{Modeling Individual Participants' Data}

As a final test of the claim that free recall effects arise from individual differences in strategies rather than core memory processes, we attempt to fit individual participant data using a model of episodic memory that does not include a strategy component. Most existing models of free recall assume that the SPC, PFR, and CRP functions are produced by fundamental memory processes. Thus, modelers have focused on identifying and modeling those processes, but generally do not model strategic elements of the task. The ability of a strategy-free model to capture free recall data is in principle an excellent way of determining whether strategies are a necessary part of accounting for free recall effects: If a strategy-free model can fit the effects, there is no need to posit strategic elements. However, most previous modeling work does not provide such a decisive test because the models generally have been fit to averaged data. It is possible that a strategy-free model can capture averaged data but fail to capture individual participants' data (Unsworth et al., 2011). Therefore, we attempt to model individual participants' data with the CMR model (Polyn et al., 2009), a model that has successfully accounted for a range of free recall effects including those considered here.

Before discussing the simulations, we provide an outline of the CRM model (for a full formal description of the model, see Polyn et al., 2009). In CMR, two types of cognitive representations interact: (a) the feature representation $(F)$, in which the features of the current list item are activated, and (b) the context representation $(C)$, in which the current state of context is activated. Hebbian associative matrices connect these representations: One connecting features to context $\left(M^{F C}\right)$ and one connecting context to features $\left(M^{C F}\right)$. Each association matrix is a weighted sum of a preexperimental component that reflects long-standing semantic relation- 

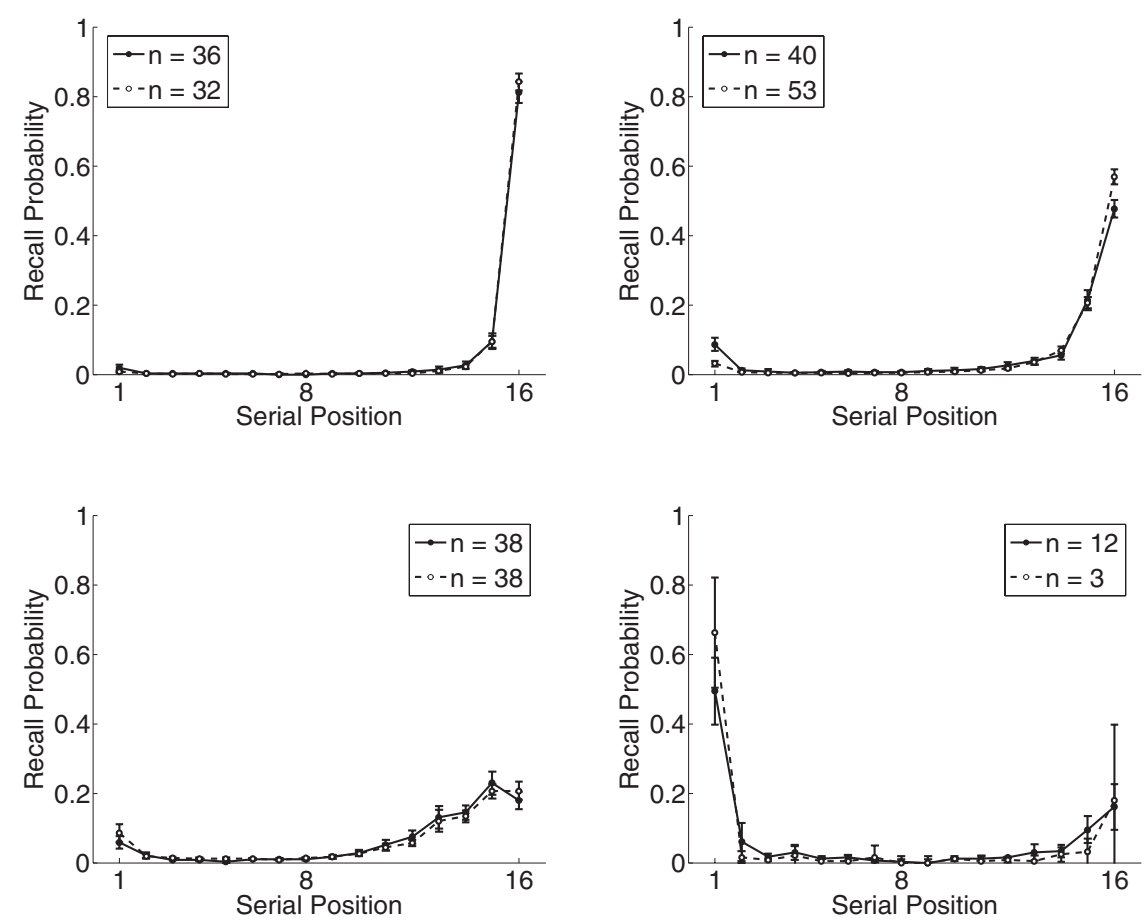

Figure 9. Means of clusters for probability of first recall functions. $K$-means with $k=4$ was used to assign each participant to a cluster. Solid lines show the no-task lists; dashed lines show the task lists. The legends give the number of participants assigned to each cluster.

ships (derived with LSA) and an experimental component that reflects new learning that occurs during the experiment.

In Figure 13, the words boat, cat, and apple have already been studied and the next word, $\operatorname{dog}$, has just been presented. Studying $d o g$ activates the corresponding features, $\mathbf{f}_{i}$, which in turn retrieve the context states to which $d o g$ has previously been associated: $\mathbf{c}^{\mathbf{I N}}{ }_{i}=M^{F C} \mathbf{f}_{i}$. This retrieved context, $\mathbf{c}^{\mathbf{I N}}{ }_{i}$, is incorporated into the context representation according to $\mathbf{c}_{i}=\rho_{i} \mathbf{c}_{i-1}+\beta \mathbf{c}^{\mathbf{I N}}{ }_{i}$. Here $\beta$ is a model parameter governing how quickly context changes $\left(\rho_{i}\right.$ is chosen such that $\left|\mathbf{c}_{i}\right|=1$ ).

During recall, the current contextual state is used to cue retrieval via the $M^{C F}$ associations: $\mathbf{f}^{\mathbf{I N}}{ }_{i}=M^{C F} \mathbf{c}_{i}$. The resulting $\mathbf{f}^{\mathbf{I N}}{ }_{i}$ gives the degree of support for each item in the model's vocabulary. This vector of support values is used as the starting point for a set of competitive accumulators, one for each word, according to the leaky competitive accumulator model of Usher and McClelland (2001). The first word to accumulate enough activation to cross a threshold is recalled. When an item wins the recall competition, its representation is reactivated on $F$, allowing the model to retrieve the contextual state associated with the item. Context is updated with the same mechanism used during the study period (although the rate of context updating $\beta$ can differ between study and recall). This updated state of context activates a different set of features (a new $\mathbf{f}^{\mathbf{I N}}$ ), and another recall competition begins. This series of competitions continues until the end of the recall period is reached, at which point the next trial begins.

Critically, under CMR all the free recall effects derive from memory processes that are assumed to be qualitatively invariant across healthy adults. Recency occurs because the context at the end of the list, which is used as a retrieval cue, most closely matches items presented near the end of the list. Contiguity occurs because each recalled item retrieves a new context that is similar to the contexts associated with its neighbors in the list and less similar to its more distant neighbors. Semantic proximity occurs because recalled items retrieve a context representation that is similar to the contexts associated with its semantic associates.

To prevent overfitting noise in participants' curves, we fit the model to the reduced curves described in the previous section (for the PFR, which was not considered in the previous section, the reduced curve consisted of Position 1; the average of Positions 2-13; and each of Positions 14, 15, and 16). For each participant, we used a genetic algorithm to minimize the root-mean-square deviation (RMSD) between model simulations and the participants' data across these 30 data points. The no-task and task lists were fit independently. ${ }^{4}$

The resulting fits are shown by the dotted lines in Figures 2-5. A visual inspection indicates that the model fits quite well for

\footnotetext{
${ }^{4}$ The fitting algorithm began by simulating 2,500,000 parameter sets randomly distributed throughout the parameter space. Then, for each participant, we calculated the fit of each of the known parameter sets to their data. We selected the best fitting parameter set from each orthant (the multidimensional equivalent of a quadrant) of the parameter space. This resulted in 2,048 parameter sets. We then added the next 2,952 best fitting sets with the constraint that they did not lie within half an orthant of any already included set. The resulting 5,000 parameter sets then served as the starting point for a genetic algorithm that ran for 30 generations, gradually decreasing the population size and increasing the number of times each parameter set was rerun, allowing it to converge efficiently on the best fitting set.
} 

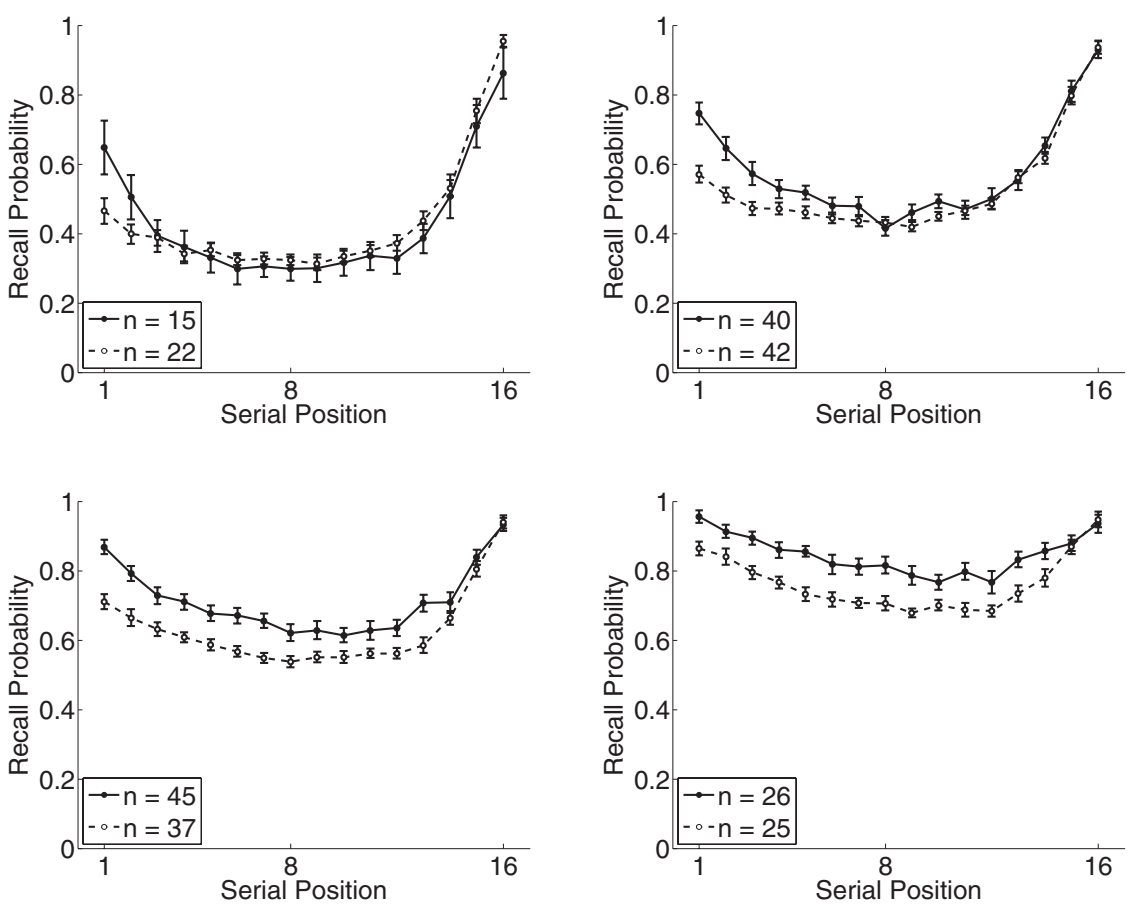

Figure 10. Means of clusters for serial position curves. $K$-means with $k=4$ was used to assign each participant to a cluster. Solid lines show the no-task lists; dashed lines show the task lists. The legends give the number of participants assigned to each cluster.

almost all the participants. To test whether the model provided an adequate fit for a given participant and a given curve, we tested whether the deviation between the best fitting model curve and the participant's curve was greater than would be expected had the two curves been drawn from the same distribution. In principle, we could use the same Mahalanobis distance test we used above when testing whether individual participants deviated from the average functions. However, the Mahalanobis test requires computing covariances, and seven sessions per participant is too few to provide stable estimates of covariance. Therefore, we adopted a slightly different procedure that does not require covariances. Specifically, for a given participant and a given curve, we computed an RMSD, $\mathrm{RMSD}_{\text {model }}$, for the deviation between model and data. We then created 100,000 simulated curves with the same means and standard deviations as the participant's data (computed across sessions). For each simulated curve we calculated an RMSD for the deviation between the simulated curve and the participant's actual curve, providing us with a distribution of $\mathrm{RMSD}_{\text {data }}$ values for curves drawn from the same distribution. The model was said to provide an adequate fit if $\mathrm{RMSD}_{\text {model }}$ fell below the 95th percentile of the $\mathrm{RMSD}_{\text {data }}$ distribution.

Participants for whom the model failed to provide an adequate fit are designated by an $M$ in their panel in Figures 2-5. For SPC, lag-CRP, and semantic-CRP, the number of poorly-fit subjects was below the $5 \%$ false detection rate at $0 \%, 0 \%$, and $0 \%$, respectively $(0.79 \%, 0 \%$, and $0 \%$ for task lists). For PFR, the model failed to fit $4.8 \%$ ( $11 \%$ for task lists) of participants. These poorly fit PFRs tended to be from participants in the chunking cluster, which suggests that these participants' initiation patterns may reflect strategy use. It is notable, however, that CMR captures the initi- ation pattern of most participants in the beginning-first cluster, which suggests that this pattern may reflect a difference in the parameterization of memory processes rather than differences in strategies (we return to this claim in the Discussion). Overall, CMR captures the recall initiation of most participants, and after recall initiation, CMR captures the recall dynamics of essentially all participants.

\section{Discussion}

Laboratory tasks designed to measure specific cognitive processes are a staple of psychological research and have provided much of the empirical foundation of our science. For this prominent position to be justified, we must be sure that the key measures derived from such tasks are not contaminated by differences across individuals in idiosyncratic strategy use (Hintzman, 2011). Here we have taken up this challenge for free recall, a task that has played a key role in memory research and theory development. Contrary to predictions of a strategy-difference account of free recall, serial position functions, lag-CRP functions, and semanticCRP functions showed no evidence of subgroups of participants employing different strategies. The average PFR curve accurately described the modal participant but disguised two qualitatively different subgroups. These results suggest that free recall effects, especially those describing postinitiation dynamics, are not substantially contaminated by different participants employing different strategies. We hope that the approach we have taken here can serve as a model for assessing the influence of strategy on important paradigms in other research areas. 

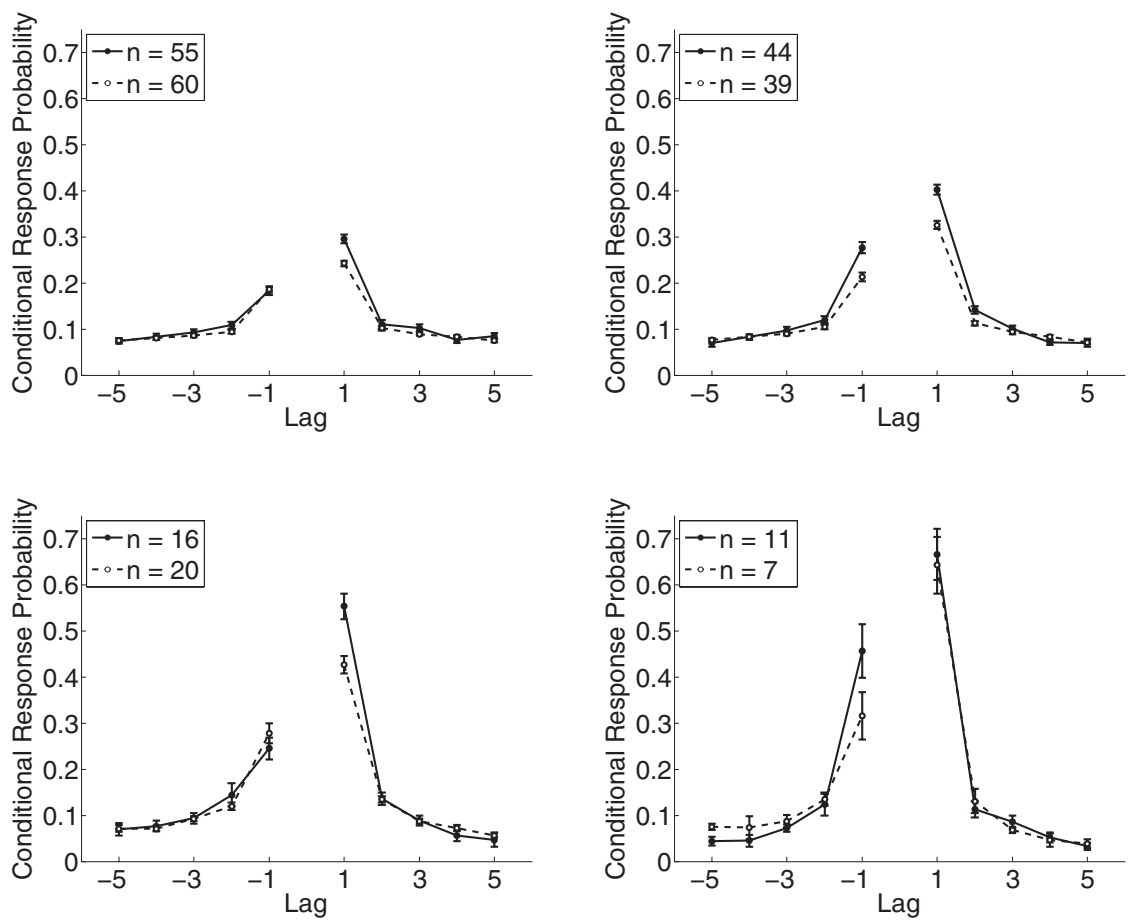

Figure 11. Means of clusters for lag-conditional response probability functions. $K$-means with $k=4$ was used to assign each participant to a cluster. Solid lines show the no-task lists; dashed lines show the task lists. The legends give the number of participants assigned to each cluster.

\section{Stability and Variability in Free Recall}

Serial position functions, lag-CRP functions, and semantic-CRP functions were all extremely consistent across participants and did not show the multimodal distributions one would expect with strategy differences. These data provided absolutely no evidence that the average function distorts individual participants' behavior. Instead the functions of most participants could be characterized as a sample from a multivariate distribution described by the average function - that is, generated by the same underlying processes. A model of these underlying processes, CMR, provided a very good fit to individual participants. In the case of temporal contiguity and semantic proximity, 126 out of 126 participants showed the effect. This level of consistency suggests that temporal contiguity and semantic proximity are universal principles in the sense that every healthy adult shows them. Indeed, the contiguity and proximity effects were so consistent across individuals that one is tempted to rename them contiguity and proximity laws.

Most participants' PFR functions were flat through early list items and began a steep, monotonic increase a few items back from the end of the list, mirroring the average function. However, we found evidence of two subgroups that showed patterns quite different from the average PFR. The first subgroup showed a "beginning-first" pattern reminiscent of the average initiation pattern seen on early trials (Dallett, 1963; Goodwin, 1976; Hasher, 1973; Huang, 1986) and with short lists (Grenfell-Essam \& Ward, 2012). The second subgroup showed a tendency to initiate recall from a variety of positions from the second half of the list rather than the strong tendency to initiate at the last item. Notably, the PFR of this subgroup is nonmonotonic, peaking about one item back from the end of the list (see Figure 9D), suggesting that items are chunked and recalled in forward order within chunks (Farrell, 2012).

\section{Strategies Versus Processes}

As detailed in the introduction, we view task performance as an interaction between task-specific strategy selection and domaingeneral memory processes. Under this view strategy plays a important role in memory tasks; it allows a common set of memory processes to be intelligently deployed to meet the demands of a wide range of tasks. Moreover, there is no doubt that there are differences between participants in the strategies that they adopt. Our analyses show, however, regardless of any variation among participants in strategy use, that there is a set of effects that are highly consistent across participants, which we suggest reflect the operation of a common set of memory processes.

Throughout the article we have built a case that differences among participants in strategy use cannot account for the shape of average free recall functions, and suggested that these curves instead represent the operation of core memory processes. Of course, the fact that differences in strategy use do not account for the shape of the curves does not logically preclude the possibility that all participants adopt a common strategy, and that the common strategy determines the shape of the curves. For example, the constraints of the task may force all participants into a common, but still ad hoc, strategy that tells us little about the memory system. Such a common-strategy account borders on unfalsifiable if we limit ourselves to examining behavioral performance on a single task (indeed one could argue that almost any effect in 

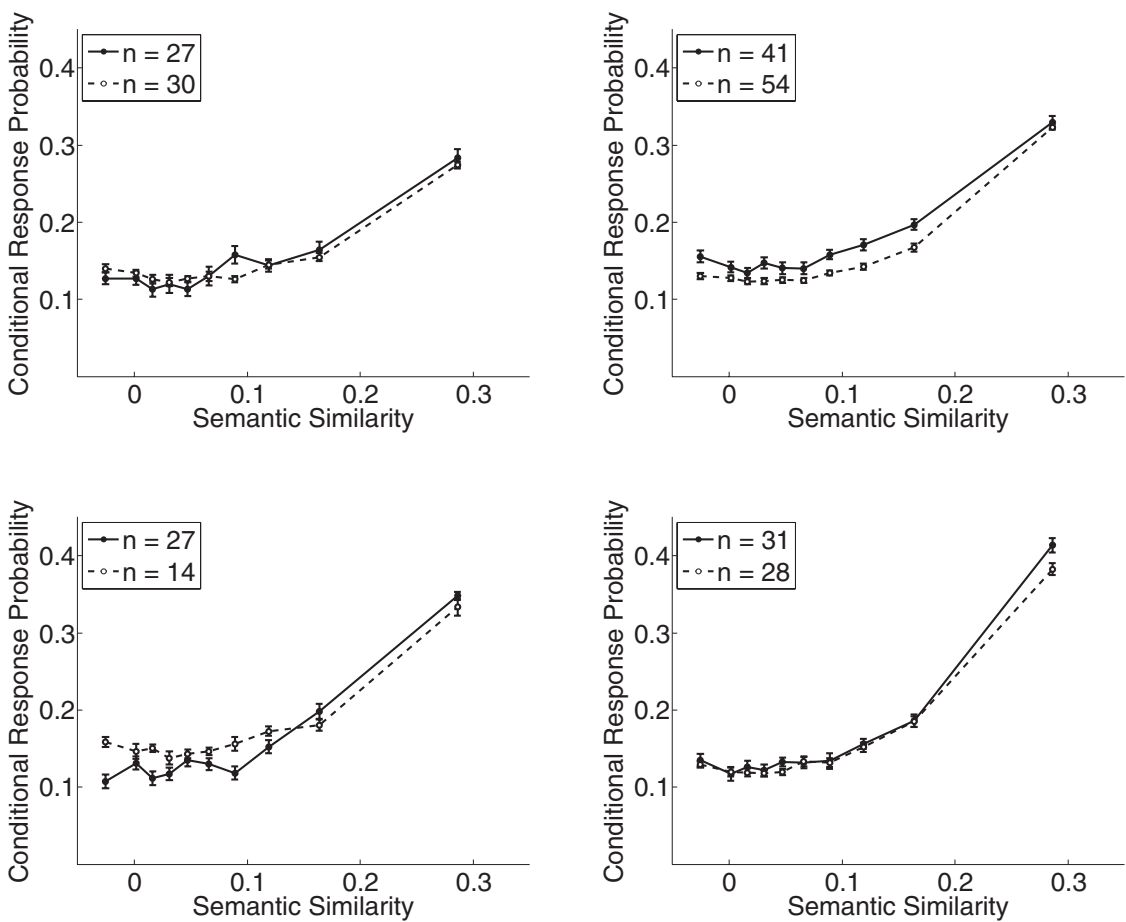

Figure 12. Means of clusters for semantic-conditional probability functions. $K$-means with $k=4$ was used to assign each participant to a cluster. Solid lines show the no-task lists; dashed lines show the task lists. The legends give the number of participants assigned to each cluster.

psychology represents a strategy rather than a cognitive process). Luckily we can gain some traction in separating common-strategy and core-process accounts in a few ways. First, we can resort to models of the cognitive system. We have shown that CMR, a model of core memory processes that has been used to account for a wide range of empirical findings without simulating strategy use, can account for the data of almost all individuals. These simulations show conclusively that the effects can be accounted for without strategies. Second, we can attempt to generalize the effects beyond a single task. The contiguity effect is observed on a variety of other tasks that do not obviously have the same strategy affordances as free recall, such as high-confidence old/new recognition responses (Schwartz et al., 2005), intrusions in paired-associate recall (Davis et al., 2008), and more naturalistic recall of autobiographical events (Moreton \& Ward, 2010).

Table 1

Correlations (Spearman's $\rho$ ) Between Mahalanobis Distances for the Four Free Recall Functions

\begin{tabular}{lllll}
\hline \multicolumn{1}{c}{ Variable } & 1 & 2 & 3 & 4 \\
\hline 1. SPC & - & & & \\
2. PFR & .11 & $\overline{.2}$ & - & \\
3. Lag-CRP & .11 & .03 & $\mathbf{. 2 6}$ & - \\
4. Semantic-CRP & .029 & .03
\end{tabular}

Note. Correlations in bold are significant at $\alpha=.05 . \mathrm{SPC}=$ serial position curve; PFR $=$ probability of first recall; $\mathrm{CRP}=$ conditional response probability.
The claim that core cognitive processes are not hopelessly obscured by strategy use should not be surprising: A good strategy must operate within the constraints of how the memory system operates. For example, a strategy that ignores the powerful effect of semantic proximity on the memorability of items is a bad strategy. This view suggests that when participants are exposed to a new task, they likely explore different strategies, abandoning ineffective ones as they gain practice, and converging on those that take best advantage of core memory processes. Therefore, a first hurdle in attributing an effect to a core memory process is showing that it does not disappear with practice. We cleared that hurdle by examining free recall functions in participants who are highly practiced with the free recall task having completed many trials over multiple sessions. In typical single-session laboratory studies, early trials constitute a much higher percentage of the total number trials and therefore likely confound learning-to-learn effects with underlying memory processes.

However, even in the case of learning a new task, we should not overestimate the importance of strategy. If we view task performance as reflecting both a strategy component and a memory component, there is no reason to assume that only the strategy component is optimized with practice. Just as participants will explore different strategies when first exposed to a task, participants likely go through a "tuning period" in which they attempt to optimize the parameters of their memory system to fit the demands of the task. To use CMR as an example, participants may tune the parameters that control the weighting of pre-experimental semantic associations versus newly learned temporal associations to optimally balance the influence of the two types associations in 


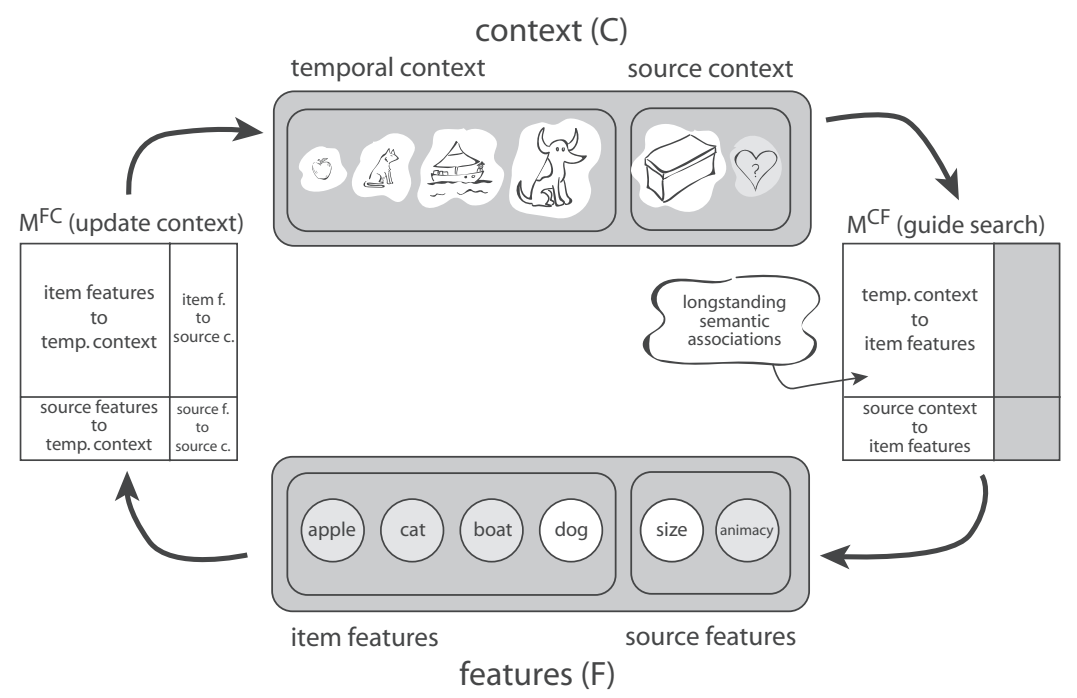

Figure 13. Schematic of context maintenance and retrieval. When an item is studied, a set of features $(F)$ is activated, corresponding to the item's identity. This set of features is then used to update a context representation $(C)$ by projecting through the feature-to-context associative weight matrix $\left(M^{F C}\right)$. Hebbian learning creates links between coactive features and context elements (on both $M^{F C}$ and $M^{C F}$ ). During recall, context is used to reactivate the features of studied items by projecting through $M^{C F}$.

guiding recall. Tuning parameters is not the same as exploring strategies; two participants may have the same strategy but different parameter settings. The distinction can be clarified by asking whether the effect in question could be captured by varying the parameters of a strategy-free model. If so, it may be a case of parameter tuning; if not, strategy differences may be the cause. For example, a weakening of semantic clustering and a strengthening of temporal clustering with repeated exposure to the same list (Klein, Addis, \& Kahana, 2005) could naturally be modeled by gradually increasing the CMR parameter that controls the weighting of new temporal associations. By contrast, the difference introduced by asking participants to encode deeply or shallowly would likely require some addition to the model to account for selective attention to stimuli features.

This logic suggests that the beginning-first and end-first recall initiation clusters do not necessarily reflect a difference in strategy. The fact that CMR can fit the beginning-first cluster suggests that it is in fact a difference in parameter tuning, as one would expect CMR to be unable to fit individuals who are using different strategies. A similar analysis could be applied to the recently reported finding that beginning-first initiation becomes less likely as list length increases (Grenfell-Essam \& Ward, 2012). Such an effect could occur because different list lengths encourage the use of different strategies, but it could also result from a strategy-free model under which both a primacy gradient and a recency gradient influence the accessibility of list items. If we assume that both primacy and recency decay with temporal separation between an item and the retrieval cue, then, all else being equal, we expect participants to initiate with an item from near the end of the list. If, however, primacy is stronger than recency, then as list length decreases and the proximity of early items to the end of the list increases, there will be a point at which primacy overcomes the temporal-proximity advantage of recency and participants shift to a beginning-first initiation pattern. Moreover, there will also be individual differences in the shift point if there are any systematic or stochastic differences between individuals in their recency and primacy parameters.

\section{Individual Differences in Recall Dynamics}

Although there is some work on individual differences in strategy selection (Bailey et al., 2008; Coyle et al., 1998), the notion of parameter tuning has received little attention. Thus, the extent to which differences between individuals on early trials are due to strategy exploration versus parameter tuning should be an important target for individual differences researchers. For example, some variables, such as orthographic distinctiveness of list items, can influence and possibly eliminate the contiguity effect (McDaniel et al., 2011) among participants with limited practice, but it remains to be seen whether these variables influence contiguity directly by disrupting memory processes or indirectly by encouraging participants to explore strategies that obscure contiguity.

Similarly, analyses reported by Unsworth et al. (2011) could be seen as showing an effect of individual differences in strategy on the shape of the SPC. They found three distinct clusters of participants, which they labeled "recency," "primacy," and "both." However, the participants completed only 10 free recall lists (compared with 112 lists for the participants reported here), and therefore the subgroups may represent variation in strategy exploration and parameter tuning and not variation in core processes. Moreover, Unsworth et al. reported results only from dividing the data into three clusters and did not report parsimony-corrected fit values for other numbers of clusters. Indeed, inspecting the SPCs of these clusters suggests that they vary quantitatively rather than qualitatively; each group seems to show both a recency and a 
primacy effect but to varying degrees. Therefore, we argue that their findings are consistent with our own.

The analyses presented here suggest that individual differences in SPC, PFR, lag-CRP, and semantic-CRP functions are largely due to quantitative variations in the efficiency of the same underlying memory processes, rather than qualitative differences in strategies employed. The claim that individual differences are quantitative rather than qualitative should not be mistaken for the claim that individual differences are unimportant. Indeed, we think individual differences have been neglected in memory research in general, and free recall research in particular. By contrast, researchers investigating working memory have a long and productive tradition of studying individual variation in memory processes and the relation of those processes to other aspects of cognition (e.g., Hasher, Lustig, \& Zacks, 2007; Kane, Poole, Tuholski, \& Engle, 2006; McCabe, Roediger, McDaniel, Balota, \& Hambrick, 2010; Miyake et al., 2000).

There is growing interest in individual differences in long-term memory tasks (Mogle, Lovett, Stawski, \& Sliwinski, 2008; Unsworth \& Engle, 2007) driven by accumulating evidence that the tasks used to study working memory actually measure, at least partly, long-term memory (Healey \& Miyake, 2009; Unsworth \& Engle, 2006). We have recently shown that four sources of variation underlie individual differences in recall dynamics, corresponding to primacy, recency, and temporal and semantic contiguity (Healey, Crutchley, \& Kahana, 2013). We expect that the emerging literature on individual differences in long-term memory will have a major impact on the field, but it is important to understand the nature of those individual differences: Quantitative variation in a common set of memory processes.

\section{Conclusions}

Laboratory paradigms have been the most important tools in developing an understanding of human cognition. Concerns that the key measures provided by these paradigms do not reflect core cognitive processes, but are in fact artifacts of averaging across individuals who employ different strategies (e.g., Hintzman, 2011), must be taken seriously. But the solution is not to abandon existing paradigms. Rather, the solution is to rigorously test their validity. Here we found that four key free recall measures are highly consistent across individuals, with only recall initiation showing signs of qualitative differences among individuals. This level of consistency, and the fact that individual participants' data are well characterized by a computational model of core memory processes that makes no use of strategy, suggest that these free recall effects reflect fundamental principles of the memory system. We hope that the analytical techniques we have introduced here can be used test the validity of other key laboratory paradigms.

\section{References}

Anderson, J. R., Bothell, D., Byrne, M. D., Douglass, S., Lebiere, C., \& Qin, Y. (2004). An integrated theory of the mind. Psychological Review, 111, 1036-1060. doi:10.1037/0033-295X.111.4.1036

Atkinson, R. C., \& Shiffrin, R. M. (1968). Human memory: A proposed system and its control processes. In K. W. Spence \& J. T. Spence (Eds.), The psychology of learning and motivation (Vol. 2, pp. 89-195). New York: Academic Press. doi:10.1016/S0079-7421(08)60422-3

Bailey, H., Dunlosky, J., \& Kane, M. J. (2008). Why does working memory span predict complex cognition? Testing the strategy affor- dance hypothesis. Memory \& Cognition, 36, 1383-1390. doi:10.3758/ MC.36.8.1383

Balota, D. A., Yap, M. J., Cortese, M. J., Hutchison, K. A., Kessler, B., Loftis, B., . . Treiman, R. (2007). The English Lexicon Project. Behavior Research Methods, 39, 445-459. doi:10.3758/BF03193014

Bousfield, W. A. (1953). The occurrence of clustering in the recall of randomly arranged associates. Journal of General Psychology, 49, 229 240. doi:10.1080/00221309.1953.9710088

Bousfield, W. A., Sedgewick, C. H., \& Cohen, B. H. (1954). Certain temporal characteristics of the recall of verbal associates. American Journal of Psychology, 67, 111-118. doi:10.2307/1418075

Bower, G. H. (1972). Stimulus-sampling theory of encoding variability. In A. W. Melton \& E. Martin (Eds.), Coding processes in human memory (pp. 85-121). New York, NY: Wiley.

Bridge, D. (2006). Memory and cognition: What difference does gender make? Unpublished honors thesis, Syracuse University.

Carstensen, L. L., Isaacowitz, D. M., \& Charles, S. T. (1999). Taking time seriously: A theory of socioemotional selectivity. American Psychologist, 54, 165-181. doi:10.1037/0003-066X.54.3.165

Chun, M. M., \& Wolfe, J. M. (2001). Visual attention. In E. B. Goldstein (Ed.), Blackwell handbook of perception (pp. 272-310). Oxford, England: Blackwell.

Coyle, T. R., Read, L. E., Gaultney, J. F., \& Bjorklund, D. F. (1998). Giftedness and variability in strategic processing on a multitrial memory task: Evidence for stability in gifted cognition. Learning and Individual Differences, 10, 273-290. doi:10.1016/S1041-6080(99)80123-X

Cusack, R., Lehmann, M., Veldsman, M., \& Mitchell, D. J. (2009). Encoding strategy and not visual working memory capacity correlates with intelligence. Psychonomic Bulletin \& Review, 16, 641-647. doi: 10.3758/PBR.16.4.641

Dalezman, J. (1976). Effects of output order on immediate, delayed, and final recall performance. Journal of Experimental Psychology: Human Learning and Memory, 2, 597-608. doi:10.1037/0278-7393.2.5.597

Dallett, K. M. (1963). Practice effects in free and ordered recall. Journal of Experimental Psychology, 66, 65-71. doi:10.1037/h0043223

Davelaar, E. J., Goshen-Gottstein, Y., Ashkenazi, A., Haarmann, H. J., \& Usher, M. (2005). The demise of short-term memory revisited: Empirical and computational investigations of recency effects. Psychological Review, 112, 3-42. doi:10.1037/0033-295X.112.1.3

Davis, O. C., Geller, A. S., Rizzuto, D. S., \& Kahana, M. J. (2008). Temporal associative processes revealed by intrusions in pairedassociate recall. Psychonomic Bulletin \& Review, 15, 64-69. doi: 10.3758/PBR.15.1.64

Deese, J., \& Kaufman, R. A. (1957). Serial effects in recall of unorganized and sequentially organized verbal material. Journal of Experimental Psychology, 54, 180-187. doi:10.1037/h0040536

Delaney, P. F., \& Knowles, M. E. (2005). Encoding strategy changes and spacing effects in the free recall of unmixed lists. Journal of Memory and Language, 52, 120-130. doi:10.1016/j.jml.2004.09.002

Delaney, P. F., Spirgel, A. S., \& Toppino, T. C. (2012). A deeper analysis of the spacing effect after "deep" encoding. Memory \& Cognition, 40, 1003-1015. doi:10.3758/s13421-012-0207-3

Duncan, J. (2010). The multiple-demand (MD) system of the primate brain: Mental programs for intelligent behaviour. Trends in Cognitive Sciences, 14, 172-179. doi:10.1016/j.tics.2010.01.004

Dunlosky, J., \& Hertzog, C. (1998). Aging and deficits in associative memory: What is the role of strategy production? Psychology and Aging, 13, 597-607. doi:10.1037/0882-7974.13.4.597

Dunlosky, J., \& Hertzog, C. (2000). Updating knowledge about encoding strategies: A componential analysis of learning about strategy effectiveness from task experience. Psychology and Aging, 15, 462-474. doi: 10.1037/0882-7974.15.3.462 
Ericsson, K. A., \& Kintsch, W. (1995). Long-term working memory. Psychological Review, 102, 211-245. doi:10.1037/0033-295X.102.2 .211

Estes, W. K. (1955). Statistical theory of spontaneous recovery and regression. Psychological Review, 62, 145-154. doi:10.1037/h0048509

Farrell, S. (2012). Temporal clustering and sequencing in short-term memory and episodic memory. Psychological Review, 119, 223-271. doi: $10.1037 / \mathrm{a} 0027371$

Garrido, L. E., Abad, F. J., \& Ponsoda, V. (2012). A new look at Horn's parallel analysis with ordinal variables. Psychological Methods. Advance online publication. doi:10.1037/a0030005

Gigerenzer, G. (2008). Why heuristics work. Perspectives on Psychological Science, 3, 20-29. doi:10.1111/j.1745-6916.2008.00058.x

Gigerenzer, G., Hoffrage, U., \& Goldstein, D. G. (2008). Fast and frugal heuristics are plausible models of cognition: Reply to Dougherty, Franco-Watkins, and Thomas (2008). Psychological Review, 115, 230239. doi:10.1037/0033-295X.115.1.230

Glanzer, M., \& Cunitz, A. R. (1966). Two storage mechanisms in free recall. Journal of Verbal Learning and Verbal Behavior, 5, 351-360. doi:10.1016/S0022-5371(66)80044-0

Goodwin, J. (1976). Changes in primacy and recency with practice in single-trial free recall. Journal of Verbal Learning and Verbal Behavior, 15, 119-132. doi:10.1016/S0022-5371(76)90012-8

Grenfell-Essam, R., \& Ward, G. (2012). Examining the relationship between free recall and immediate serial recall: The role of list length, strategy use, and test expectancy. Journal of Memory and Language, 67, 106-148. doi:10.1016/j.jml.2012.04.004

Hartwig, M. K., \& Dunlosky, J. (2012). Study strategies of college students: Are self-testing and scheduling related to achievement? Psychonomic Bulletin \& Review, 19, 126-134. doi:10.3758/s13423-011-0181-y

Hasher, L. (1973). Position effects in free recall. American Journal of Psychology, 86, 389-397. doi:10.2307/1421449

Hasher, L., Lustig, C., \& Zacks, R. (2007). Inhibitory mechanisms and the control of attention. In A. R. A. Conway, C. Jarrold, M. J. Kane, A. Miyake, \& J. N. Towse (Eds.), Variation in working memory (pp. 227-249). New York, NY: Oxford University Press.

Healey, M. K., Crutchley, P., \& Kahana, M. J. (2013). Individual differences in memory search and their relation to intelligence. Manuscript submitted for publication.

Healey, M. K., \& Miyake, A. (2009). The role of attention during retrieval in working-memory span: A dual-task study. Quarterly Journal of Experimental Psychology, 62, 733-745.

Hintzman, D. L. (2011). Research strategy in the study of memory: Fads, fallacies, and the search for the "coordinates of truth". Perspectives on Psychological Science, 6, 253-271. doi:10.1177/1745691611406924

Hogan, R. M. (1975). Interitem encoding and directed search in free recall. Memory \& Cognition, 3, 197-209. doi:10.3758/BF03212898

Howard, M. W., Jing, B., Addis, K. M., \& Kahana, M. J. (2007). Semantic structure and episodic memory. In T. K. Landauer, D. S. McNamara, S. Dennis, \& W. Kintsch (Eds.), Handbook of latent semantic analysis (pp. 121-141). Mahwah, NJ: Erlbaum.

Howard, M. W., \& Kahana, M. J. (1999). Contextual variability and serial position effects in free recall. Journal of Experimental Psychology: Learning, Memory, and Cognition, 25, 923-941. doi:10.1037/0278-7393 .25 .4 .923

Howard, M. W., \& Kahana, M. J. (2002). When does semantic similarity help episodic retrieval? Journal of Memory and Language, 46, 85-98. doi:10.1006/jmla.2001.2798

Huang, I. (1986). Transitory changes of primacy and recency in successive single-trial free recall. Journal of General Psychology, 113, 5-21. doi: 10.1080/00221309.1986.9710539

Jenkins, J. J. (1979). Four points to remember: Tetrahedral model of memory experiments. In L. S. Cermak \& F. I. M. Craik (Eds.), Levels of processing in human memory (pp. 429-446). Hillsdale, NJ: Erlbaum.
Kahana, M. J. (1996). Associative retrieval processes in free recall. Memory \& Cognition, 24, 103-109. doi:10.3758/BF03197276

Kahana, M. J. (2012). Foundations of human memory. New York, NY Oxford University Press.

Kahana, M. J., \& Howard, M. W. (2005). Spacing and lag effects in free recall of pure lists. Psychonomic Bulletin \& Review, 12, 159-164. doi:10.3758/BF03196362

Kahana, M. J., Howard, M. W., \& Polyn, S. M. (2008). Associative retrieval processes in episodic memory. In H. L. Roediger III (Ed.), Learning and memory: A comprehensive reference: Vol. 2. Cognitive psychology of memory (pp. 1-24). Oxford, England: Elsevier.

Kahana, M. J., Howard, M. W., Zaromb, F., \& Wingfield, A. (2002). Age dissociates recency and lag recency effects in free recall. Journal of Experimental Psychology: Learning, Memory, and Cognition, 28, 530540. doi:10.1037/0278-7393.28.3.530

Kahneman, D., \& Wright, P. (1971). Changes of pupil size and rehearsal strategies in a short-term memory task. Quarterly Journal of Experimental Psychology, 23, 187-196. doi:10.1080/14640747108400239

Kane, M. J., Poole, B. J., Tuholski, S. W., \& Engle, R. W. (2006). Working memory capacity and the top-down control of visual search: Exploring the boundaries of "executive attention". Journal of Experimental Psychology: Learning, Memory, and Cognition, 32, 749-777. doi:10.1037/ 0278-7393.32.4.749

Klein, K. A., Addis, K. M., \& Kahana, M. J. (2005). A comparative analysis of serial and free recall. Memory \& Cognition, 33, 833-839. doi:10.3758/BF03193078

Laming, D. (1999). Testing the idea of distinct storage mechanisms in memory. International Journal of Psychology, 34, 419-426. doi 10.1080/002075999399774

Laming, D. (2006). Predicting free recalls. Journal of Experimental Psychology: Learning, Memory, and Cognition, 32, 1146-1163. doi 10.1037/0278-7393.32.5.1146

Laming, D. (2008). An improved algorithm for predicting free recalls. Cognitive Psychology, 57, 179-219. doi:10.1016/j.cogpsych.2008.01 .001

Landauer, T. K., \& Dumais, S. T. (1997). A solution to Plato's problem: The latent semantic analysis theory of acquisition, induction, and representation of knowledge. Psychological Review, 104, 211-240. doi: 10.1037/0033-295X.104.2.211

Loewenstein, G. F., \& Prelec, D. (1993). Preferences for sequences of outcomes. Psychological Review, 100, 91-108. doi:10.1037/0033-295X .100 .1 .91

Loftus, G. R., \& Masson, M. E. J. (1994). Using confidence intervals in within-subject designs. Psychonomic Bulletin \& Review, 1, 476-490. doi:10.3758/BF03210951

Marewski, J. N., \& Schooler, L. J. (2011). Cognitive niches: An ecological model of strategy selection. Psychological Review, 118, 393-437. doi: 10.1037/a0024143

Marshall, P. H., \& Werder, P. R. (1972). The effects of the elimination of rehearsal on primacy and recency. Journal of Verbal Learning and Verbal Behavior, 11, 649-653. doi:10.1016/S0022-5371(72)80049-5

Mata, R., Schooler, L. J., \& Rieskamp, J. (2007). The aging decision maker: Cognitive aging and the adaptive selection of decision strategies Psychology and Aging, 22, 796-810. doi:10.1037/0882-7974.22.4.796

McCabe, D. P., Roediger, H. L., III, McDaniel, M. A., Balota, D. A., \& Hambrick, D. Z. (2010). The relationship between working memory capacity and executive functioning: Evidence for a common executive attention construct. Neuropsychology, 24, 222-243. doi:10.1037/ a0017619

McDaniel, M. A., \& Bugg, J. M. (2008). Instability in memory phenomena: A common puzzle and a unifying explanation. Psychonomic Bulletin \& Review, 15, 237-255. doi:10.1037/a0017619

McDaniel, M. A., Cahill, M., Bugg, J. M., \& Meadow, N. G. (2011). Dissociative effects of orthographic distinctiveness in pure and mixed 
lists: An item-order account. Memory \& Cognition, 39, 1162-1173. doi:10.3758/s13421-011-0097-9

McGeoch, J. A. (1932). Forgetting and the law of disuse. Psychological Review, 39, 352-370. doi:10.1037/h0069819

Miyake, A., Friedman, N., Emerson, M., Witzki, A., Howerter, A., \& Wager, T. (2000). The unity and diversity of executive functions and their contributions to complex "frontal lobe" tasks: A latent variable analysis. Cognitive Psychology, 41, 49-100. doi:10.1006/cogp.1999 .0734

Modigliani, V., \& Hedges, D. G. (1987). Distributed rehearsals and the primacy effect in single-trial free recall. Journal of Experimental Psychology: Learning, Memory, and Cognition, 13, 426-436. doi:10.1037/ 0278-7393.13.3.426

Mogle, J. A., Lovett, B. J., Stawski, R. S., \& Sliwinski, M. J. (2008). What's so special about working memory? An examination of the relationships among working memory, secondary memory, and fluid intelligence. Psychological Science, 19, 1071-1077. doi:10.1111/j.14679280.2008.02202.x

Moreton, B. J., \& Ward, G. (2010). Time scale similarity and long-term memory for autobiographical events. Psychonomic Bulletin \& Review, 17, 510-515. doi:10.3758/PBR.17.4.510

Moscovitch, M., \& Winocur, G. (2002). The frontal cortex and working with memory. In D. T. Stuss \& R. T. Knight (Eds.), Principles of frontal lobe function (pp. 188-209). New York, NY: Oxford University Press. doi:10.1093/acprof:oso/9780195134971.003.0012

Murdock, B. B. (1962). The serial position effect of free recall. Journal of Experimental Psychology, 64, 482-488. doi:10.1037/h0045106

Murdock, B. B., \& Metcalfe, J. (1978). Controlled rehearsal in single-trial free recall. Journal of Verbal Learning and Verbal Behavior, 17, 309324. doi:10.1016/S0022-5371(78)90201-3

Murdock, B. B., \& Walker, K. D. (1969). Modality effects in free recall. Journal of Verbal Learning and Verbal Behavior, 8, 665-676. doi: 10.1016/S0022-5371(69)80120-9

Paivio, A., \& Yuille, J. C. (1969). Changes in associative strategies and paired-associate learning over trials as a function of work imagery and type of learning set. Journal of Experimental Psychology, 79, 458-463. doi: $10.1037 / \mathrm{h} 0026929$

Payne, J. W., Bettman, J. R., \& Johnson, E. J. (1988). Adaptive strategy selection in decision making. Journal of Experimental Psychology: Learning, Memory, and Cognition, 14, 534-552. doi:10.1037/02787393.14.3.534

Pelleg, D., \& Moore, A. W. (2000). X-means: Extending $k$-means with efficient estimation of the number of clusters. In P. Langley (Ed.), Proceedings of the Seventeenth International Conference on Machine Learning (pp. 727-734). San Francisco, CA: Kaufman.

Pham, D. T., Dimov, S. S., \& Nguyen, C. D. (2005). Selection of K in k-means clustering. Proceedings of the Institution of Mechanical Engineers, Part C: Journal of Mechanical Engineering Science, 219, 103119. doi:10.1243/095440605X8298

Polyn, S. M., Norman, K. A., \& Kahana, M. J. (2009). A context maintenance and retrieval model of organizational processes in free recall. Psychological Review, 116, 129-156. doi:10.1037/a0014420

Postman, L. (1969). Experimental analysis of learning to learn. In G. H. Bower \& J. T. Spence (Eds.), Psychology of learning and motivation (Vol. 3, pp. 241-297). New York, NY: Academic Press.

Postman, L., \& Phillips, L. W. (1965). Short-term temporal changes in free recall. Quarterly Journal of Experimental Psychology, 17, 132-138. doi:10.1080/17470216508416422

Rieskamp, J., \& Otto, P. E. (2006). SSL: A theory of how people learn to select strategies. Journal of Experimental Psychology: General, 135, 207-236. doi:10.1037/0096-3445.135.2.207

Roediger, H. L., III. (2008). Relativity of remembering: Why the laws of memory vanished. Annual Review of Psychology, 59, 225-254. doi: 10.1146/annurev.psych.57.102904.190139
Romney, A. K., Brewer, D. D., \& Batchelder, W. H. (1993). Predicting clustering from semantic structure. Psychological Science, 4, 28-34. doi:10.1111/j.1467-9280.1993.tb00552.x

Rundus, D. (1980). Maintenance rehearsal and long-term recency. Memory \& Cognition, 8, 226-230. doi:10.3758/BF03197610

Sahakyan, L., \& Delaney, P. F. (2003). Can encoding differences explain the benefits of directed forgetting in the list method paradigm? Journal of Memory and Language, 48, 195-206. doi:10.1016/S0749596X(02)00524-7

Schwartz, G., Howard, M. W., Jing, B., \& Kahana, M. J. (2005). Shadows of the past: Temporal retrieval effects in recognition memory. Psychological Science, 16, 898-904. doi:10.1111/j.1467-9280.2005.01634.x

Sederberg, P. B., Gauthier, L. V., Terushkin, V., Miller, J. F., Barnathan, J. A., \& Kahana, M. J. (2006). Oscillatory correlates of the primacy effect in episodic memory. NeuroImage, 32, 1422-1431. doi:10.1016/j neuroimage.2006.04.223

Sederberg, P. B., Howard, M. W., \& Kahana, M. J. (2008). A contextbased theory of recency and contiguity in free recall. Psychological Review, 115, 893-912. doi:10.1037/a0013396

Sederberg, P. B., Miller, J. F., Howard, W. H., \& Kahana, M. J. (2010). The temporal contiguity effect predicts episodic memory performance. Memory \& Cognition, 38, 689-699. doi:10.3758/MC.38.6.689

Simon, H. A. (1956). Rational choice and the structure of the environment. Psychological Review, 63, 129-138. doi:10.1037/h0042769

Solway, A., Murdock, B. B., \& Kahana, M. J. (2012). Positional and temporal clustering in serial order memory. Memory \& Cognition, 40, 177-190. doi:10.3758/s13421-011-0142-8

Steyvers, M., Shiffrin, R. M., \& Nelson, D. L. (2004). Word association spaces for predicting semantic similarity effects in episodic memory. In A. F. Healy (Ed.), Experimental cognitive psychology and its applications: Decade of behavior. Washington, DC: American Psychological Association. doi:10.1037/10895-018

Stoff, D. M., \& Eagle, M. N. (1971). The relationship among reported strategies, presentation rate, and verbal ability and their effects on free recall learning. Journal of Experimental Psychology, 87, 423-428. doi: $10.1037 / \mathrm{h} 0030541$

Sugar, C. A., \& James, G. M. (2003). Finding the number of clusters in a dataset. Journal of the American Statistical Association, 98, 750-763. doi:10.1198/016214503000000666

Tan, L., \& Ward, G. (2000). A recency-based account of the primacy effect in free recall. Journal of Experimental Psychology: Learning, Memory, and Cognition, 26, 1589-1625. doi:10.1037/0278-7393.26.6.1589

Turley-Ames, K., \& Whitfield, M. (2003). Strategy training and working memory task performance. Journal of Memory and Language, 49, 446468. doi:10.1016/S0749-596X(03)00095-0

Tversky, A., \& Kahneman, D. (1973). Availability: A heuristic for judging frequency and probability. Cognitive Psychology, 5, 207-232. doi: 10.1016/0010-0285(73)90033-9

Unsworth, N., Brewer, G., \& Spillers, G. (2011). Inter- and intra-individual variation in immediate free recall: An examination of serial position functions and recall initiation strategies. Memory, 19, 67-82. doi: $10.1080 / 09658211.2010 .535658$

Unsworth, N., \& Engle, R. (2006). Simple and complex memory spans and their relation to fluid abilities: Evidence from list-length effects. Journal of Memory and Language, 54, 68-80. doi:10.1016/j.jml.2005.06.003

Unsworth, N., \& Engle, R. (2007). The nature of individual differences in working memory capacity: Active maintenance in primary memory and controlled search from secondary memory. Psychological Review, 114, 104-132. doi:10.1037/0033-295X.114.1.104

Usher, M., \& McClelland, J. L. (2001). The time course of perceptual choice: The leaky, competing accumulator model. Psychological Review, 108, 550-592. doi:10.1037/0033-295X.108.3.550

Ward, G. (2002). A recency-based account of the list length effect in free recall. Memory \& Cognition, 30, 885-892. doi:10.3758/BF03195774 
Waugh, N. C., \& Norman, D. (1965). Primary memory. Psychological Review, 72, 89-104. doi:10.1037/h0021797

Wright, P., \& Kahneman, D. (1971). Evidence for alternative strategies of sentence retention. Quarterly Journal of Experimental Psychology, 23, 197-213. doi:10.1080/14640747108400240

Zaromb, F. M., Howard, M. W., Dolan, E. D., Sirotin, Y. B., Tully, M., Wingfield, A., \& Kahana, M. J. (2006). Temporal associations and prior-list intrusions in free recall. Journal of Experimental Psychology:
Learning, Memory, and Cognition, 32, 792-804. doi:10.1037/02787393.32.4.792

Received August 7, 2012

Revision received May 29, 2013

Accepted June 9, 2013 